\title{
DOI: https://doi.org/10.24297/jac.v18i.8948
}

Six Reasons to Discard Wave Particle Duality:

\section{Thereby Opening New Territory for Young Scientists to Explore}

\author{
Author: Jeffrey H. Boyd* \\ Affiliation: * Retired. 57 Woods Road, Bethany, CT, 06524-3103, USA \\ Jeffrey.Boyd@alumni.harvard.edu
}

\begin{abstract}
:
Wave particle duality is a cornerstone of quantum chemistry and quantum mechanics (QM). But there are experiments it cannot explain, such as a neutron interferometer experiment. If QM uses $\psi$ as its wavefunction, several experiments suggest that nature uses $-\psi$ instead. The difference between $-\psi$ and $+\psi$ is that they describe entirely different pictures of how nature is organized. For example, with $-\psi$ quantum particles follow waves backwards, which is incompatible with wave-particle-duality, obviously. We call the $-\psi$ proposal the Theory of Elementary Waves (TEW). It unlocks opportunities for young scientists with no budget to conduct the basic research for a new, unexplored science. This is a dream come true for young scientists: the discovery of uncharted territory. We show how TEW explains the double slit, Pfleegor Mandel and Davisson Germer experiments, Feynman diagrams and the Bell test experiments. We provide innovative research designs for which $-\psi$ and $+\psi$ would predict divergent outcomes. What makes QM so accurate is its probability predictions. But Born's law would yield the same probabilities if it were changed from $P=|+\Psi|^{2}$ to $P=|-\Psi|^{2}$. This article is accompanied by a lively YouTube video, "6 reasons to discard wave particle duality."
\end{abstract}

Keywords: quantum chemistry, Bi-Rays, Bell test experiments, entanglement.

\section{Introduction}

"Wave-particle-duality" means that something in the quantum world behaves like a wave when observed one way, a particle when observed another way, but these are two aspects of the same thing. For example, in a double slit experiment a particle leaves the gun, and a particle hits the target screen. But in-between it allegedly acts as if it were a wave, going through both slits, interfering with itself and causing a wave pattern on the target screen. $(4,5)$

The doctrine of wave-particle-duality is so central to quantum mechanics (QM) that anyone who questions it is suspected of being "unscientific." But a hallmark of science is that firmly held beliefs might disintegrate if we focus on empirical observations that violate the orthodox teaching.(56) In this article we will examine six experiments for which wave-particle-duality is not the best explanation. We will also give you the design for three new experiments that have never been conducted, which can answer the question which picture of nature is accurate.

We are about to demonstrate that neutrons follow waves backwards. That idea is part of a paradigm shift called the Theory of Elementary Waves (TEW), based on the premise that, if $+\psi$ is the wavefunction used in $\mathrm{QM}$, then $-\psi$ is the wavefunction used by nature.(12-40,59) QM is the most accurate and productive science of all time. But what does that mean? It means the probability predictions of QM are accurate. However, probabilities are not used in QM! It uses the square root of probabilities, called "amplitudes" or "wavefunctions." Since every probability has two square roots, is it $+\psi$ or $-\psi$ that is so accurate? This is a question that science has never addressed. The experiments cited in this article favor $-\psi$ and TEW. Because of Born's law, $P=|+\psi|^{2}=|-\psi|^{2}$, that mountain of empirical data that supports QM, could equally well support TEW, and QM could be wrong! $(10,11)$ If $-\psi$ is correct then the organization of nature is unfamiliar and needs exploring, and young scientists will be the ones to do that.

What comes into view is an entirely new approach to chemistry, physics and mathematics. It is like discovering a new continent that no one has explored. A discovery of that magnitude excites the young people, even as it horrifies the old establishment. Experiments needed to explore this are inexpensive, and often cost nothing. 
Science in the past has been obsessed with matter and energy, especially with dark matter and dark energy. Those are expensive and unrewarding avenues to pursue. Scientific leaders are not interested in an arena that lacks matter and energy. When we tell them these elementary waves have been sculpting and shaping nature forever, they don't listen. TEW offers a young person an inexpensive way to make a name for herself or himself by doing the pioneering work. This article and the accompanying YouTube video tell you where to find the trailheads to begin your journey.

\section{A Neutron Interferometer Experiment}

A neutron interferometer experiment published in 1992 by Kaiser, Clothier, Werner, et. al. produced data that cannot be explained by wave-particle-duality. Briefly, as they added more and more bismuth, all wave interference died out. But when a Nearly Perfect (NP) Analyzer Crystal of silicon was inserted downstream from the interferometer, robust sinusoidal waves emerged from the final neutron beam. It appeared as if an NP Analyzer Crystal downstream restored vigorous wave interference upstream, inside the interferometer.(55,69)

The researchers explained this by saying that the NP Analyzer Crystal brought a strong sinusoidal wave out of an apparently "incoherent" neutron beam (i.e. a flat line). With the Analyzer Crystal "we picked out of the 'incoherent' beam a certain portion of the neutrons that still yields contrast. The analyzer thus restores the contrast ...." The crystal increased the coherence length of a neutron wave packet from 86 to $3450 \AA$. The data indicate that wave-packets don't represent actual neutrons. "The neutron wave-packet formalism is merely a mathematical description of Wheeler's 'great smoky dragon," they wrote. $(55,69)$

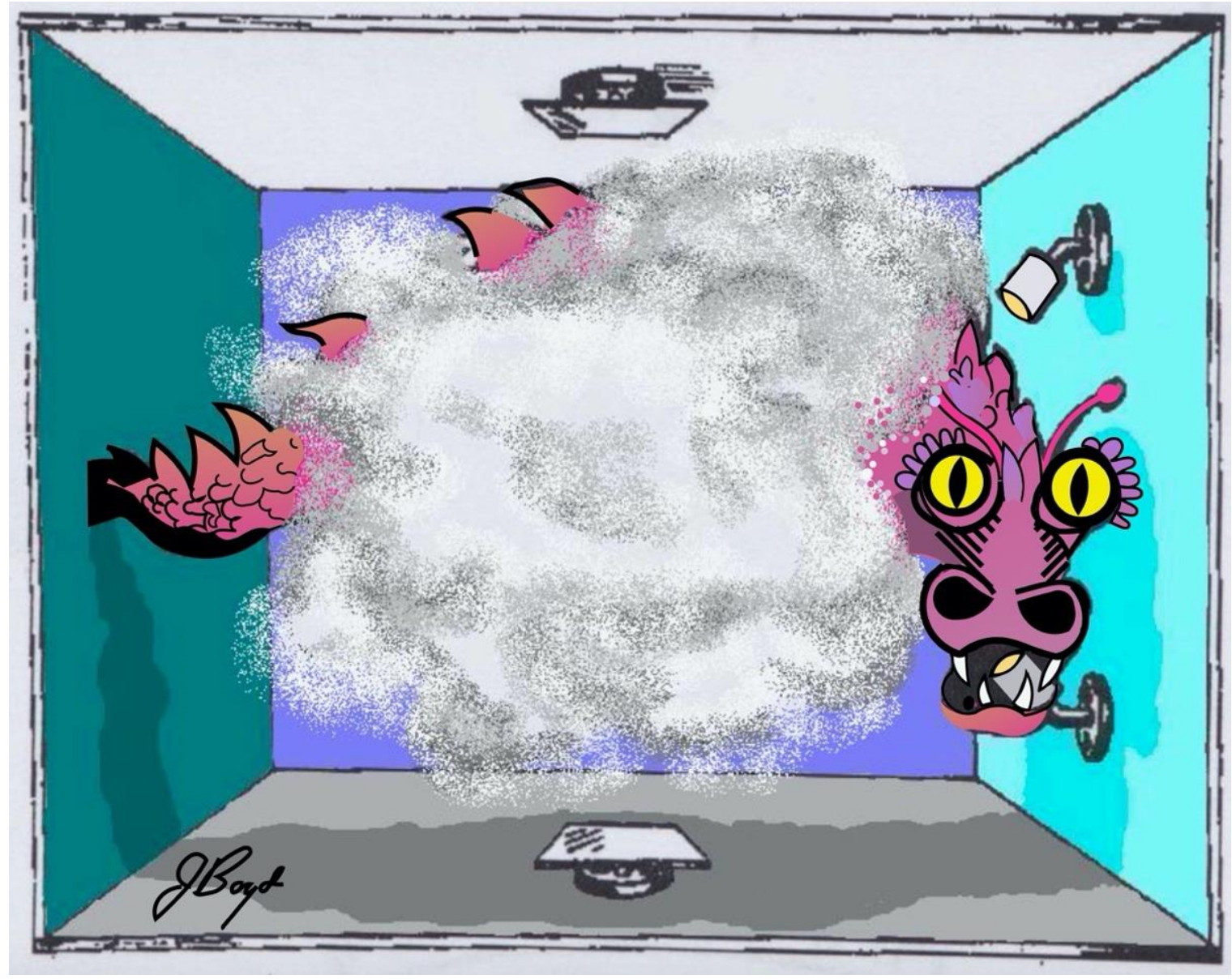

Fig. 1. Kaiser, et. al, cited this cartoon of "Wheeler's great smoky dragon." The original text says, "Smoky dragon as symbol of Bohr's elementary quantum phenomenon." We added color and simplified the cloud of smoke, but otherwise this is the cartoon cited by Kaiser as the equivalent of quantum math. (Fig. 3, p. 142 (61))

Let's ponder the unthinkable. What if we allowed ourselves to think that a robust sinusoidal curve in the interferogram, means there is robust interference inside the interferometer? That is what an interferogram 
normally measures. Fig. 2 shows what the data are telling us. The presence or absence of the NP Analyzer Crystal determines the presence or absence of robust interference inside the interferometer. This defines a cause-andeffect relationship. Fig. 2 would only make sense if the NP Analyzer Crystal were upstream from the interference!

\section{With $20 \mathrm{~mm}$ of bismuth}

NP Analyzer Crystal Present Absent

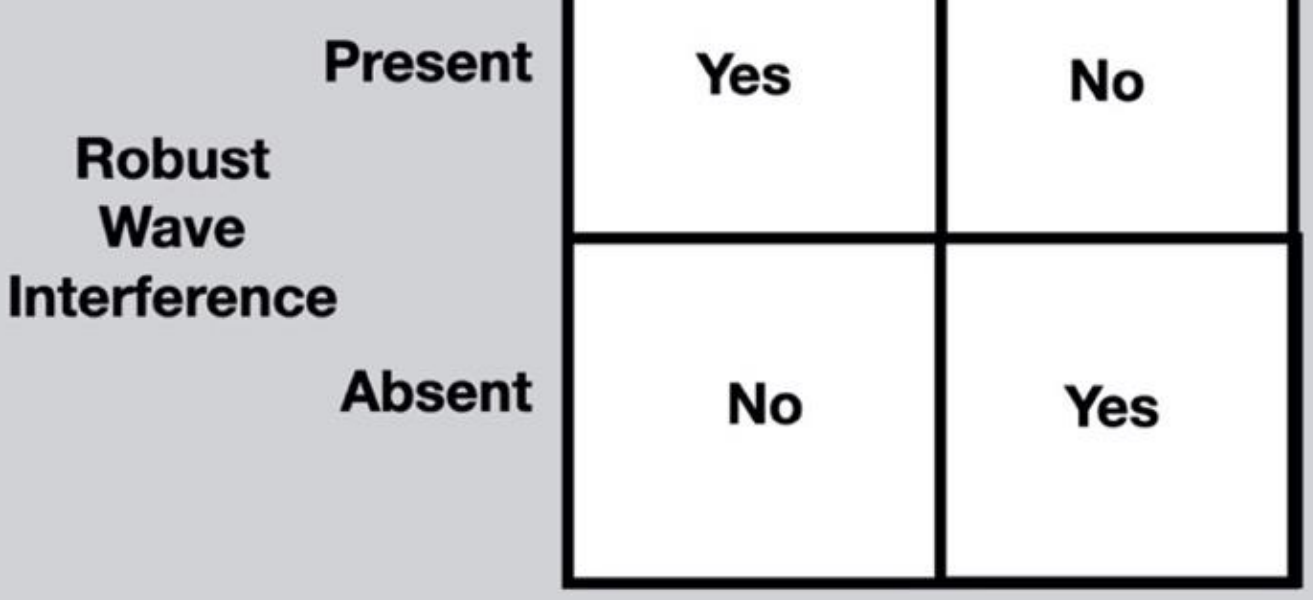

Fig. 2. The analyzer crystal and robust wave interference are each present if and only if the other is present. This is what the data actually say. This establishes a cause-and-effect relationship.

That is difficult to ponder because it is contradicts the assumption that neutron waves and neutron particles travel in the same direction. This peculiar idea never occurred to the researchers, presumably because it made no sense. When this author phoned Helmut Kaiser to discuss that, we had a friendly conversation. He spoke of Bragg interference. I spoke of wave direction. We didn't understand each other. We were politely puzzled and confused about what the other was talking about.

What is the next step in thinking the unthinkable? If waves were travelling in the opposite direction as neutrons, such hypothetical waves couldn't carry any energy. We will inquire about "zero-energy-waves" later. Currently we'll focus on the direction of these eerie hypothetical waves.

Although our explanation sounds illogical, it fits the experimental data better than an explanation that requires "Wheeler's great smoky dragon." Our explanation passes the Occam's Razor test.

Let's examine the neutron interferometer experiment in detail. Neutrons entering an interferometer were divided into two beams $\psi_{1}$ and $\psi_{2}$ (Fig. 3). At the bifurcation an oscillating aluminum plate induced a oscillating phase difference between $\psi_{1}$ and $\psi_{2}$, so there was wave interference when the two beams merged later. Therefore, the detector outside the interferometer saw a sinusoidal wave pattern. $(55,69)$ 


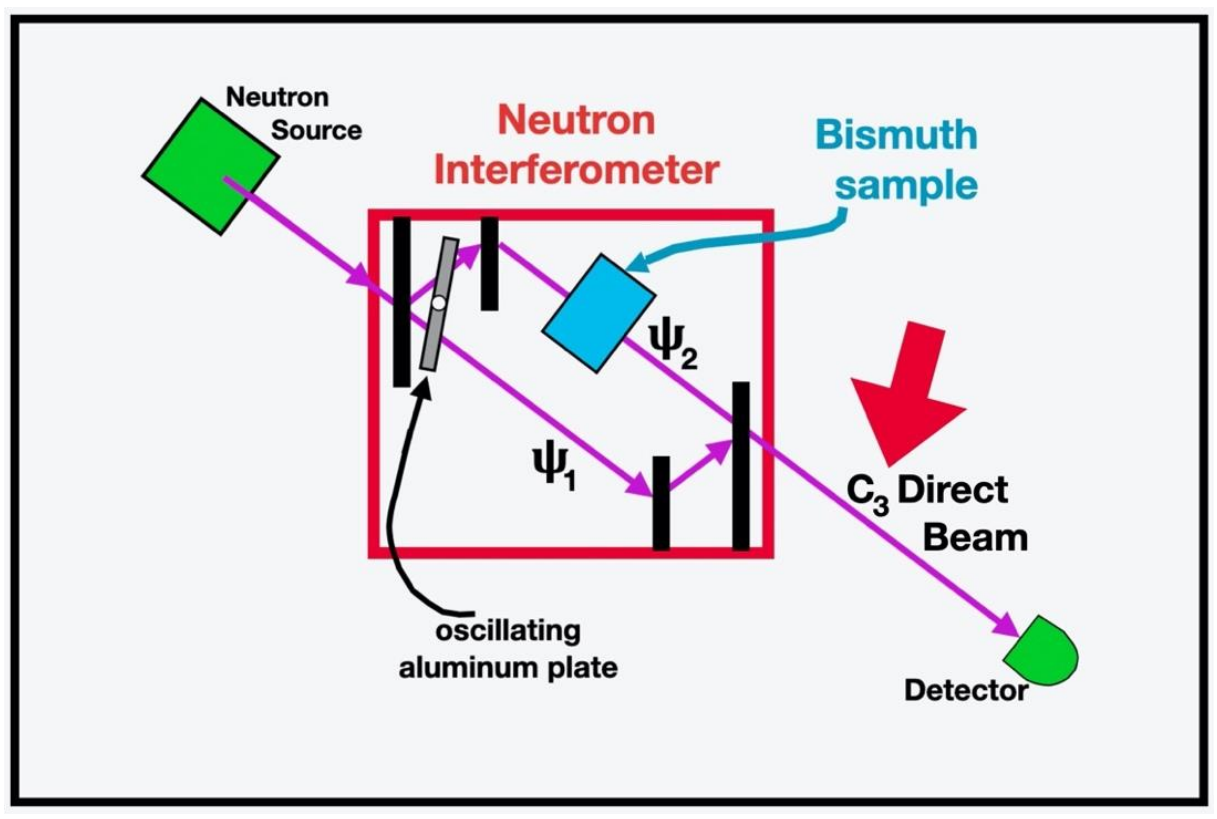

Fig. 3. Kaiser et.al. sent a beam of neutrons into an interferometer, where silicon blades (in black) divided it into $\psi_{1}$ and $\psi_{2}$ which acquired an oscillating phase shift relative to one another. The $\psi_{2}$ beam passed through a bismuth sample that slowed it down. After the beams were recombined, the height of sinusoidal waves diminished as more and more bismuth was added.

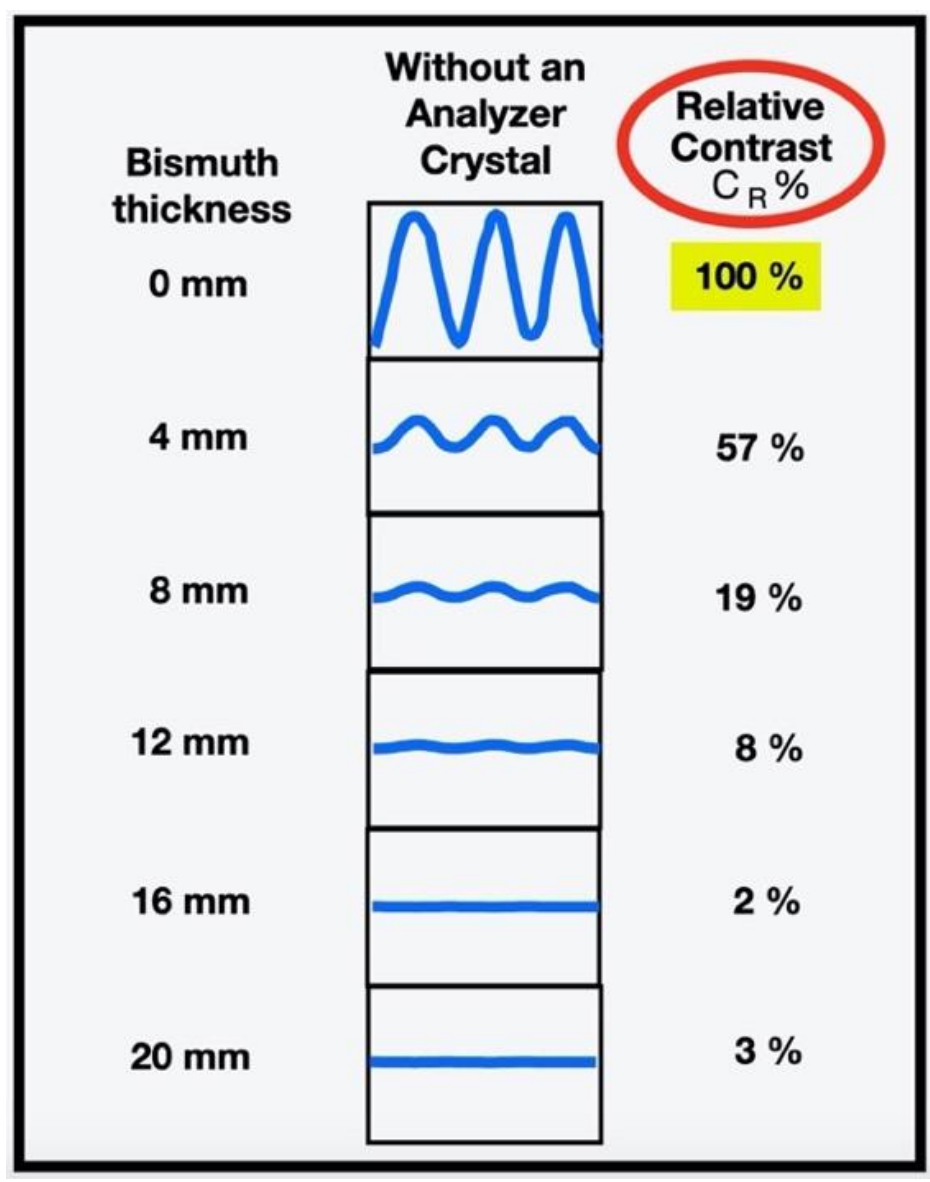

Fig. 4. This shows what "Relative Contrast $C_{R} \%$ " means. The height of the sinusoidal curve diminishes as more bismuth is added. The zero-bismuth wave is benchmarked at "100\%." 
Bismuth slows down neutrons. A neutron wave packet had a width of $86 \AA$. A maximum sample of $20 \mathrm{~mm}$ of Bismuth delayed that wave packet by $435 \AA$. A sample of bismuth was placed in the $\psi_{2}$ beam. As more and more bismuth was added from zero to $4 \mathrm{~mm}$ to $8 \mathrm{~mm}$ the $\psi_{2}$ wave packet was increasingly delayed so there was a diminishing amount of interference. With more than $12 \mathrm{~mm}$ of bismuth all interference disappeared (Fig. 4). Presumably the $\psi_{2}$ wave packet was so delayed that the $\psi_{1}$ wave packet exited the interferometer before the $\psi_{2}$ wave packet arrived at the reunion point.

"Relative contrast" $\left(C_{R} \%\right)$ is the variable to pay attention to. "Contrast" means the amplitude of the oscillation divided by its mean value. "Relative contrast" $\left(C_{R} \%\right)$ means the amplitude of the sinusoidal interferogram when bismuth is present, compared to the amplitude when there was no bismuth (Fig. 4).

The researchers repeated exactly the same experiment with one small difference. In front of the detector, they inserted a "Nearly Perfect" (NP) Analyzer Crystal of silicon in an antiparallel (111) configuration. At wavelength $\lambda=2.35 \AA$ the Bragg angle was $\theta_{A}=-22.0^{\circ}$ and $\Delta \theta=0.02^{\circ}$ full width at half maximum (FWHM), $\eta_{\mathrm{A}}=0.0035 \mathrm{rad}$. The crystal focused the beam, decreasing the standard deviation of wavelengths from $0.0120 \AA$ to $0.00030 \AA$, and increasing the height of the Gaussian. The beam should penetrate the detector better.

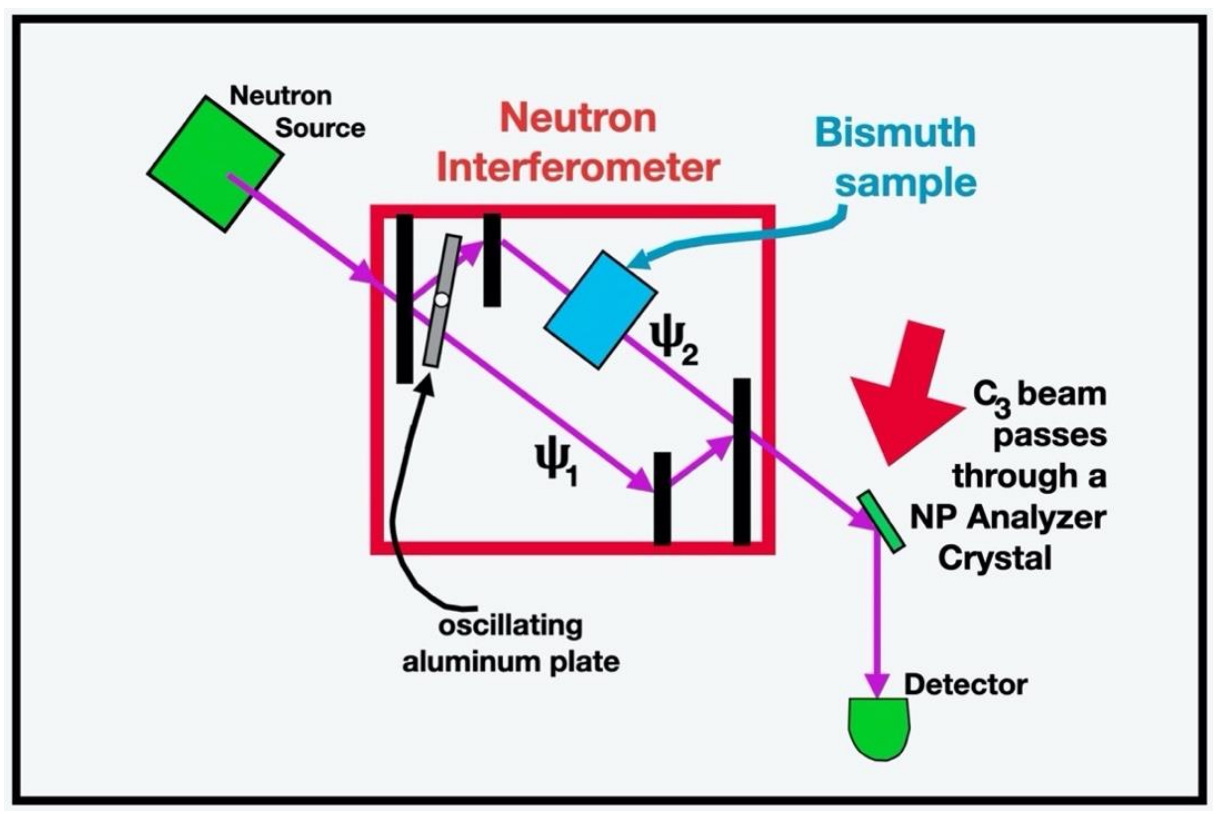

Fig. 5. They repeated exactly the same experiment with one small change: a Nearly Perfect (NP) silicon analyzer crystal was inserted before the neutron beam reached the detector.

\begin{tabular}{|c|c|c|}
\hline $\begin{array}{c}\text { Bismuth width } \\
\text { in mm }\end{array}$ & $\begin{array}{c}C_{3} \text { Direct Beam } \\
\text { Without Analyzer Crystal } \\
C_{R} \%\end{array}$ & $\begin{array}{c}C_{3} \text { Beam With } \\
\text { NP Analyzer Crystal } \\
C_{R} \%\end{array}$ \\
\hline $0 \quad \mathrm{~mm}$ & $100 \%$ & $100 \%$ \\
\hline $4.01 \mathrm{~mm}$ & $57.3 \pm 1.0$ & $97.1 \pm 5.1$ \\
\hline $12.26 \mathrm{~mm}$ & $8.0 \pm 0.8$ & $89.6 \pm 4.4$ \\
\hline $16.15 \mathrm{~mm}$ & $1.8 \pm 0.8$ & $86.0 \pm 4.8$ \\
\hline $20.08 \mathrm{~mm}$ & $2.9 \pm 0.6$ & $95.2 \pm 5.2$ \\
\hline
\end{tabular}

Table 1. The amplitude of the sinusoidal curve of the $C_{3}$ Direct exit beam without an analyzer crystal (Fig. 3), compared to the $C_{3}$ beam passing through an NP Analyzer Crystal (Fig. 5). (Data are copied from the righthand column of Tables III and VI, of Kaiser, et. al.(55)) 


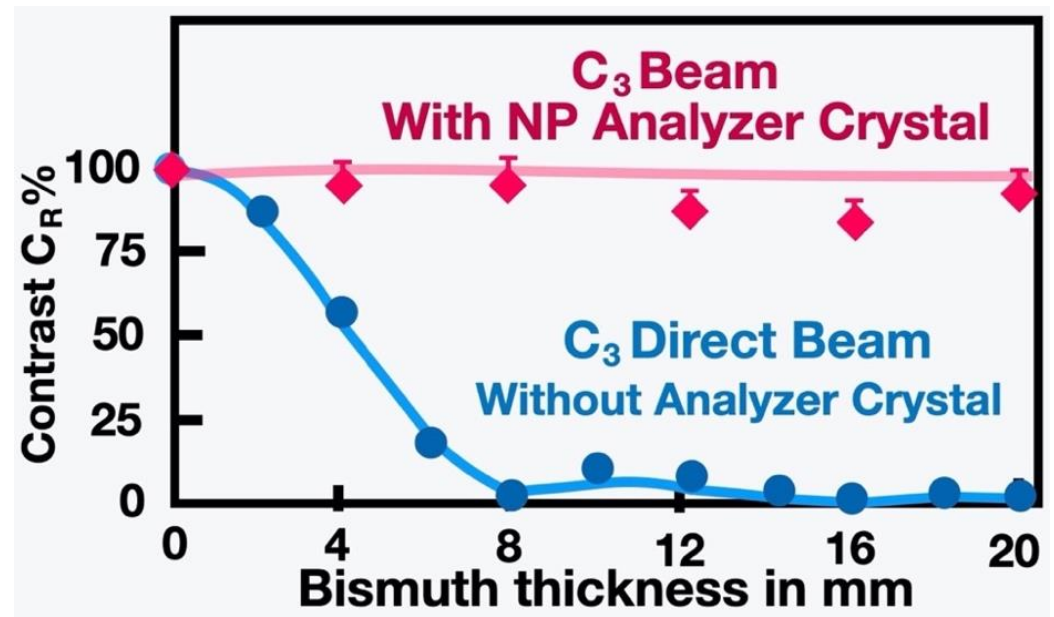

Fig. 6. The NP Analyzer Crystal restores robust interference (high $C_{R} \%$, meaning tall sinusoidal waves) inside the interferometer. (Copied from the top and bottom graphs of Fig. 9, page 40 of Kaiser, et. al.(55))

As we said, there are two ways to understand the data from Table 1 and Fig. 6. Kaiser postulated that somehow the NP Analyzer Crystal was able to conjure a strong sinusoidal wave from a flat line. They admitted this didn't make a lot of sense. They assumed there was zero wave interference. It would be akin to administering CPR that resuscitated a dead dataset: turning a flat wave into a zombie heartbeat. We say no CPR is needed. A robust interferogram indicates robust interference inside the interferometer.

\subsection{The Double Slit Experiment DISPROVES Wave Particle Duality!}

If particles follow waves backwards, then wave-particle-duality must be wrong. How do we explain the double slit experiment? Although others say that the double slit experiment proves wave-particle-duality, we claim it disproves wave-particle-duality! (22,26-27)

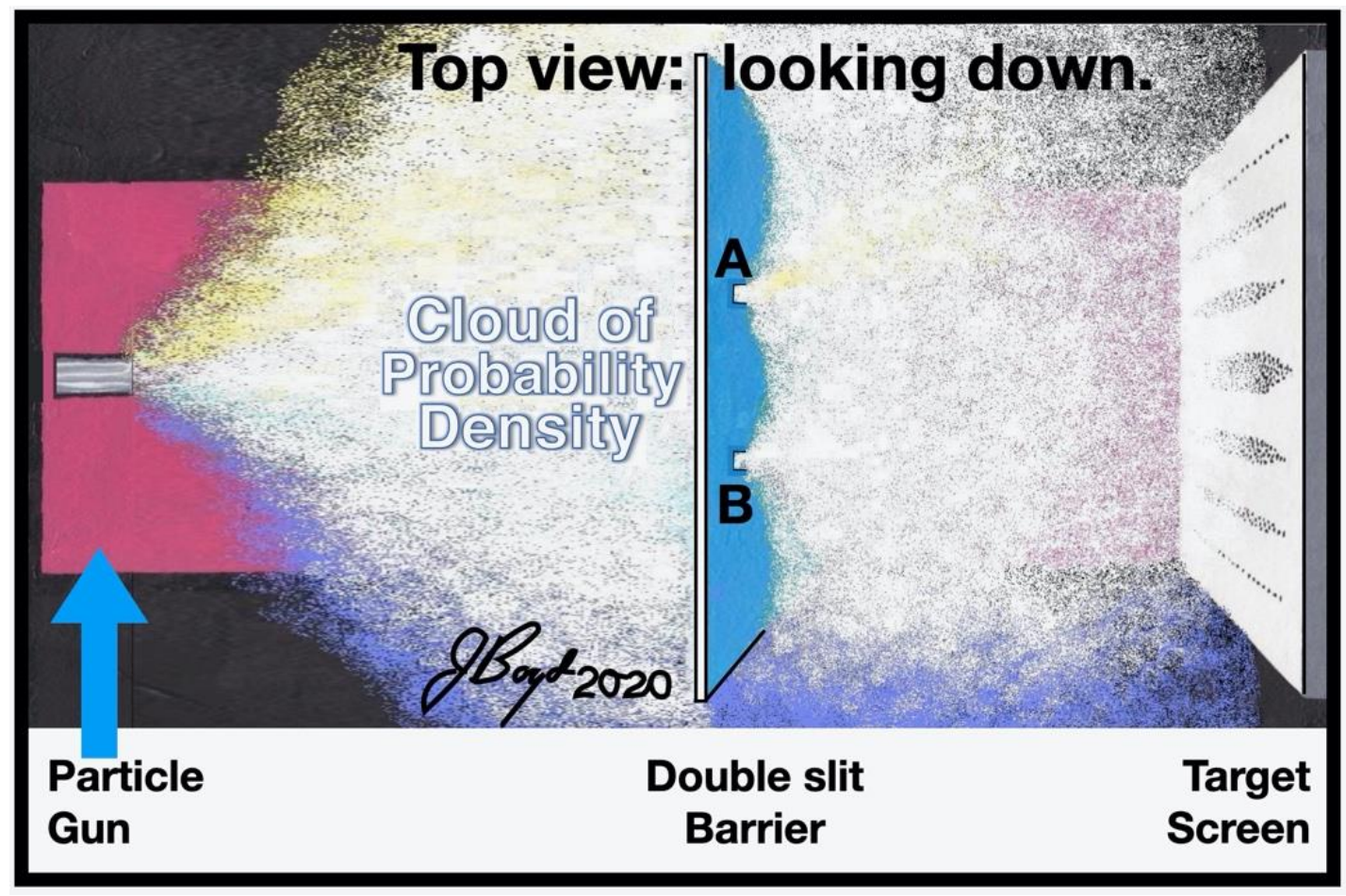

Fig. 7. The entire cloud of probability density in a double slit experiment needs to vanish instantly, faster than the speed of light, whenever a dot appears anywhere.

Consider what happens if one particle at a time is fired from the gun in Fig. 7. Then one particle at a time must register on the target screen. At that instant when a dot appears anywhere, the entire probability density cloud 
everywhere must vanish, faster than the speed of light. If any region of the cloud persists after a dot randomly appears, there would be two problems:

1. The dot would not contain $100 \%$ of the probability.

2. The persisting area of cloud could produce a second dot.

It is impossible for the entire cloud of probability density to vanish instantaneously, faster than the speed of light, whenever any dot unpredictably appears anywhere. Therefore, the conventional picture of the double slit experiment must be wrong! Nature must use some other mechanism. But what mechanism?

TEW proposes another mechanism (bottom Fig. 8). Zero-energy waves of all wavelengths emanate from every point on the target screen before a particle is emitted. We call them "elementary waves." We will limit our attention to waves of wavelength equal to the de Broglie wavelength of the particle that will be emitted later. Waves from point $\boldsymbol{\alpha}$ move toward the double slit barrier in an arc. Depending on the location of $\boldsymbol{\alpha}$ relative to midline, a wave crest arrives at slit A before, simultaneous with, or later than the same wave arrives at slit B. After penetrating the slits backwards, the two waves (from $\boldsymbol{\alpha}$ through A, versus from $\boldsymbol{\alpha}$ through B) arrive at the particle gun slightly before, simultaneous with, or later than one another. So, there is elementary wave interference converging on the gun. We picture wave interference on the proximate side of the double slit barrier, whereas your teachers have told you wave interference is on the distant side of the double slit barrier (Fig. 8).

A particle in the gun is bombarded by a zillion incoming waves (one from each point on the target screen) and chooses among them at random, in proportion to the square of the amplitude of that wave. If the emission of a particle is triggered by a wave from $\boldsymbol{\alpha}$, then the particle would follow that wave backwards with a probability of one (deterministically), through one and only one slit (it doesn't matter which slit), subject to no further wave interference, and inevitably strike point $\boldsymbol{\alpha}$ from which its wave emanates. It is easy to show that this mechanism would produce the same interference fringe pattern as QM.(22,26-27)

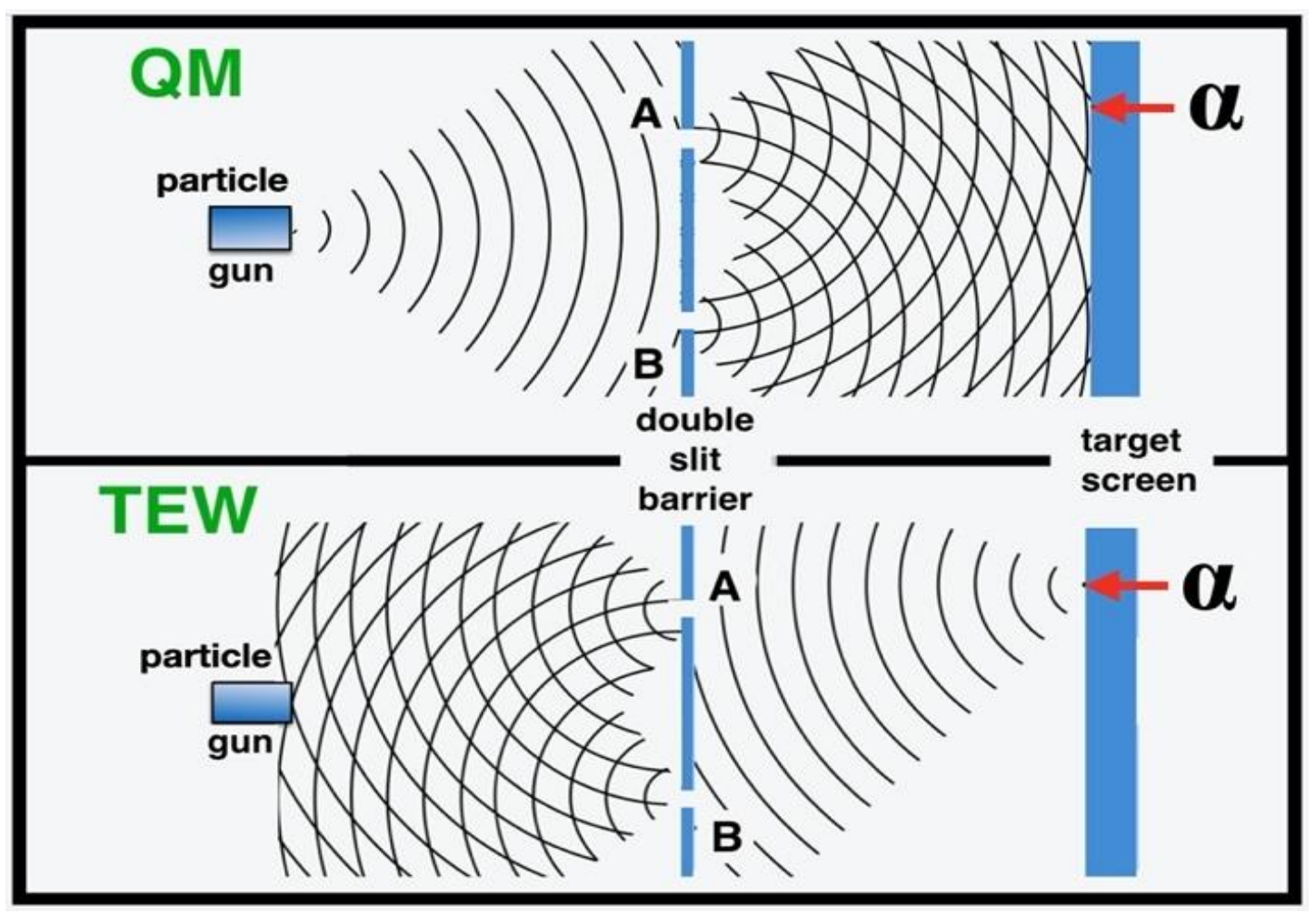

Fig. 8. The QM model of a double slit experiment compared to the TEW model with waves traveling in the opposite direction. 


\subsection{Complementarity}

"Complementarity" means that the interference pattern on the target screen vanishes if we know which slit a particle used. QM says that experimental data are different if observed than not observed. TEW asserts that complementarity is caused by the equipment, not human observation, not by human consciousness.

To know which slit a particle used, we need to introduce a light source and detector inside the equipment. Since elementary waves convey zero-energy, the energy from the lamp is infinitely greater than the zero-energy of the elementary waves. That lamp changes the elementary waves as they pass backwards through the slits, so they lose their superposition additivity. The waves from point $\boldsymbol{\alpha}$ through slit A, no longer interferes with the waves from $\boldsymbol{\alpha}$ through slit B as they converge on the gun. Their amplitudes no longer add together. We know why: because nature uses $-\psi$ rather than $+\psi$ as its wavefunction. This is confirmed empirically.

What does an interference fringe pattern on the target screen mean? It is a picture of the elementary wave interference impinging on the particle gun! If there is a lamp near one of the slits, we just said there is no such interference, and therefore no wave pattern would appear on the target screen. This explains "complementarity."

\subsection{Zero Energy Waves}

Some readers declare that all waves require energy. They say zero-energy waves could accomplish nothing.

That is a peculiar way of thinking! Schrödinger waves convey no energy. They might carry a Hamiltonian operator, but they do not transport raw energy. Neither Schrödinger nor elementary waves push nor pull particles. Neither does any work. Such waves predict how particles are likely to behave, but do not pressure the particles to behave that way. Schrödinger waves and elementary waves are the same, except they travel in opposite directions (without time reversal).

Elementary waves are unlike any other waves. At any point in space there are an infinity of elementary waves, traveling in all directions, at all wavelengths, at the speed of light, even with no particle nearby. All the energy, momentum and electromagnetism come from particles, not elementary waves. Most elementary waves involve no particle and are therefore undetectable.

\subsection{The direction of time}

Many people get confused about the direction of time in TEW. They think that if particles follow waves backwards, there must be time reversal. That is not the TEW way-of-thinking. In TEW time always goes forwards, never backwards. If an elementary wave travels eastward with time going forwards, then a particle follows that wave westward with time going forwards. If an elementary wave travels west southwest with time going forwards, then a particle follows that wave backwards in a east southeast direction with time going forwards.

TEW is not related to the time-reversal ideas of John Wheeler and Richard Feynman's essay, "Interaction with the absorber as the mechanism of radiation", nor is TEW related to John Cramer and Ruth Kastner's "transactional interpretation of QM."

The reason this is hard to think about is that we tend to think of $+\psi$ as being the wave function of nature, which implies that waves and particles travel in the same direction (unless there is time reversal). But when we switch to $-\psi$ as the wave function of nature, suddenly everything is in disarray in our mind. It changes how cause-andeffect works. It changes how we think about time. It changes everything. It is like a different universe.

One way to grasp the TEW concept of time is to read about Richard Feynman's propagator (or kernel) in section 5 below (see Equation 1). That propagator is based on the idea of integrating a function from point (a) to point (b). With TEW we define a "reverse propagator" by integrating Feynman's function from point (b) to (a). A law of mathematics is that when you reverse the bounds of integration you arrive at the negative of the original integral. All this happens with time going forwards. 
The switch from thinking that the wave function of nature is $+\psi$ to $-\psi$ being the wave function of nature, requires a drastic change in how your brain is organized. For the past century scientists have talked about how the quantum world doesn't make sense. It turns out that is because their thinking had never switched from $+\psi$ to $-\psi$. If the reader shifts gears in that way, the quantum world will look very similar to the classical world.

\section{Pfleegor and Mandel's experiment: the third reason to discard Wave Particle Duality}

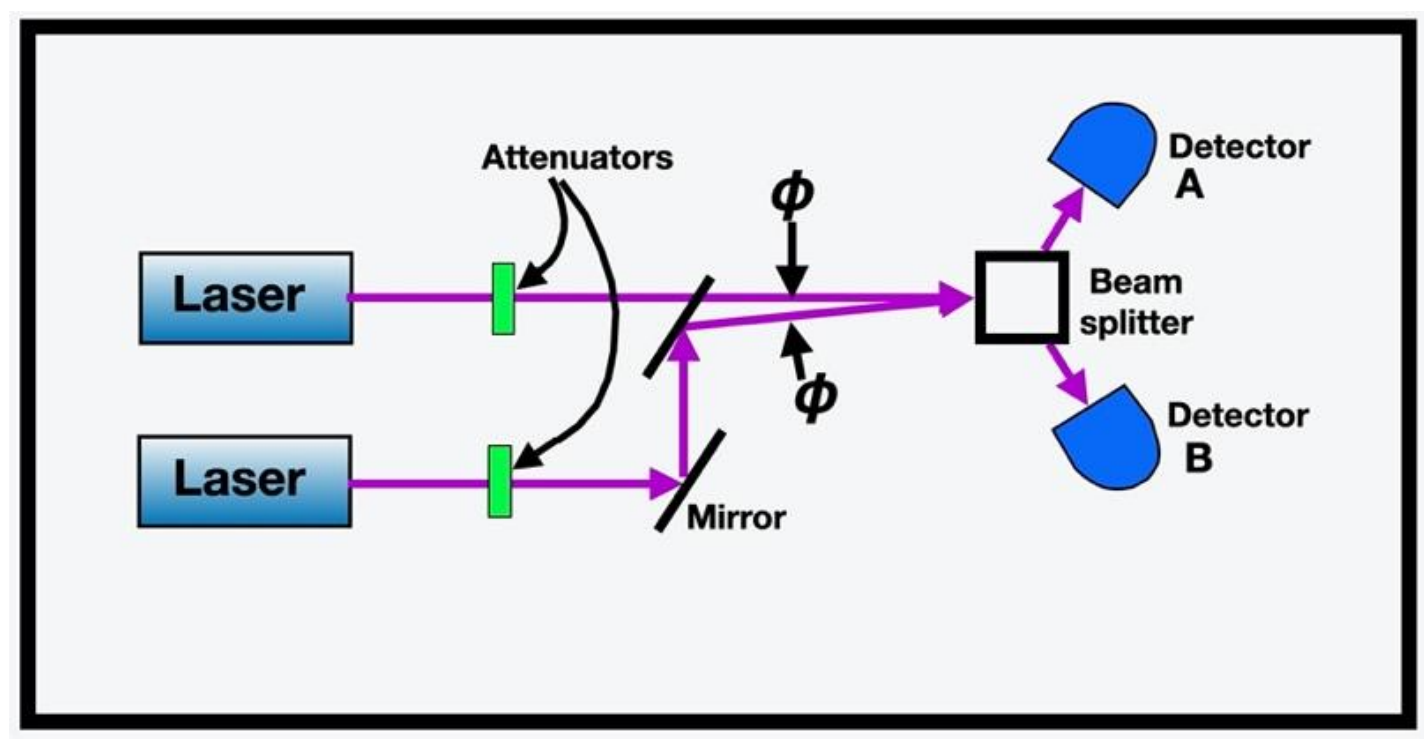

Fig. 9. Pfleegor and Mandel's experiment is similar to photons coming through two slits, but here it is two lasers. Two He-Ne laser beams overlap so they cause an interference fringe pattern. Researchers controlled $\varphi$, the angle of overlap. (Fig.1, p. 1085.(62-63))

Pfleegor and Mandel's experiment contradicts wave-particle-duality. If we fire one photon at a time, and a double slit experiment shows a wave pattern on the target screen, wave-particle-duality says each photon goes through both slits and interferes with itself. Pfleegor and Mandel conducted a different experiment using one photon at a time, but instead of two slits they used two lasers. There was an interference fringe pattern. That solitary photon cannot have come from two places to interfere with itself, because it came from one laser or the other, not both.(62-63)

It took 3 nanoseconds for each photon to cross the equipment in Fig. 9. They attenuated the laser beams to the point that every photon was followed by an average of 150 nanoseconds of empty time with no photon. There were never two photons inside the experiment. The interference fringe pattern persisted despite attenuation.

This is now the third of three experiments that wave-particle-duality cannot explain. This experiment implies that solitary photons were affected by zero-energy waves, like a kayak going down a river undulating over standing waves. Because the kayak is different than the waves, therefore wave-kayak duality is wrong. This experiment does not tell us whether the waves are traveling in the pilot wave direction, or the TEW direction.

\section{The Davisson Germer experiment is the $4^{\text {th }}$ reason to discard wave particle duality}

Most people say that the Davisson Germer experiment "proves" wave-particle-duality. We disagree.(42-44)

Davisson and Germer shot electrons at a nickel crystal and graphed the angle at which they were detected (Fig. 10). More electrons than expected registered at 54 volts (at $50^{\circ}$ ), which is what would happen if waves of wavelength $\lambda=1.67 \AA$ were diffracting through the crystal. When they published their results there was excitement about what they called the "spur" in their data.

Fig. 10 shows that most electrons weren't involved in the spur. Most electrons rebounded off the crystal at other angles and other voltages. It shows three things:

1. Evidence of electrons ricocheting like bullets (true of the majority of electrons);

2. Evidence of waves of approximately $\lambda=1.67 \AA$ wavelength, and 
3. Evidence of interaction between the particles and waves.

Since the data show those three things, why not say that, rather than claiming that electrons and waves are two aspects of the same thing, which the data do not prove?

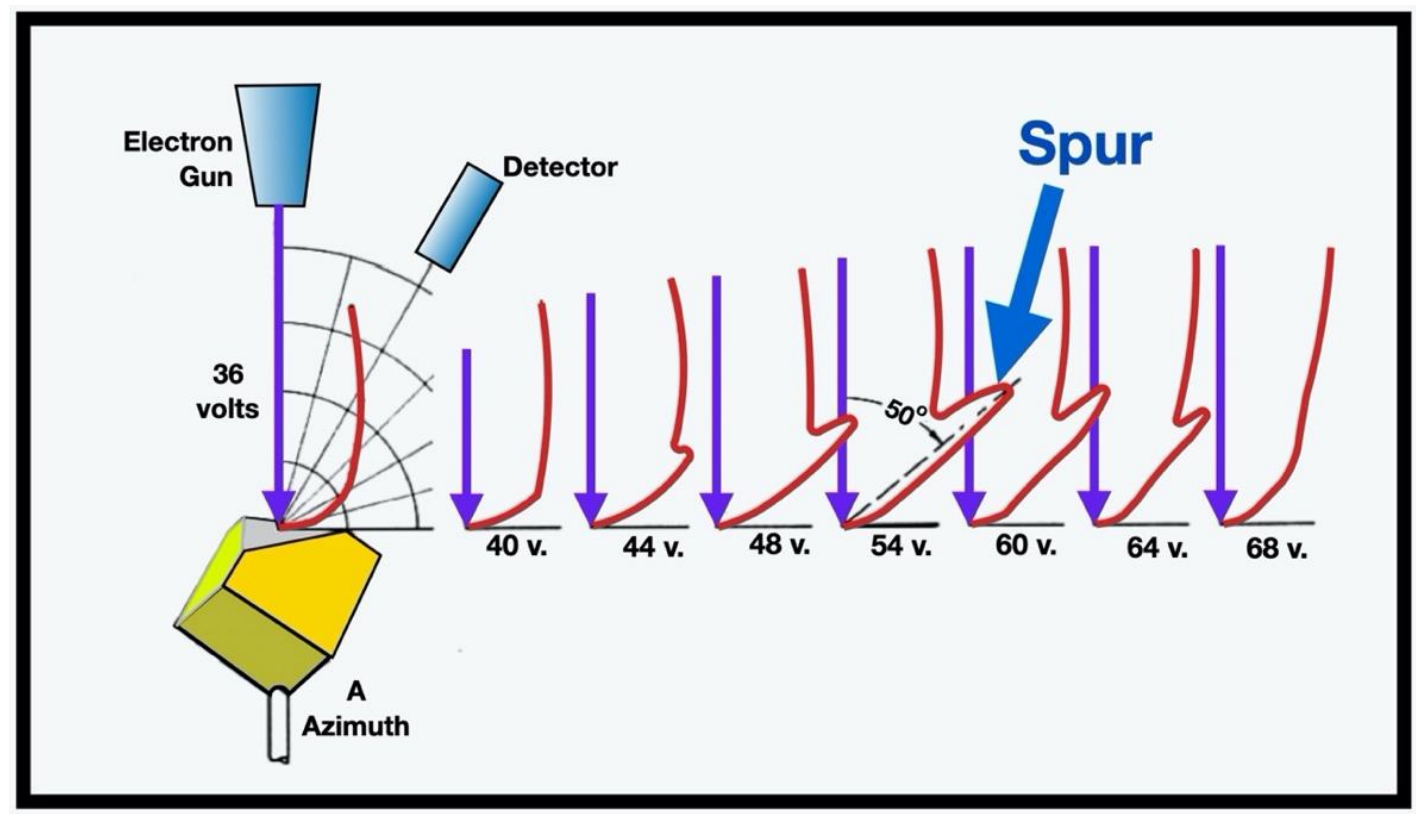

Fig. 10. Davisson and Germer fired electrons at a crystal of nickel and graphed the angle of detection. They noted an unexpected "spur", greatest at 54 volts and $50^{\circ}$. (Copied from Fig. 3, p. 95 (43))

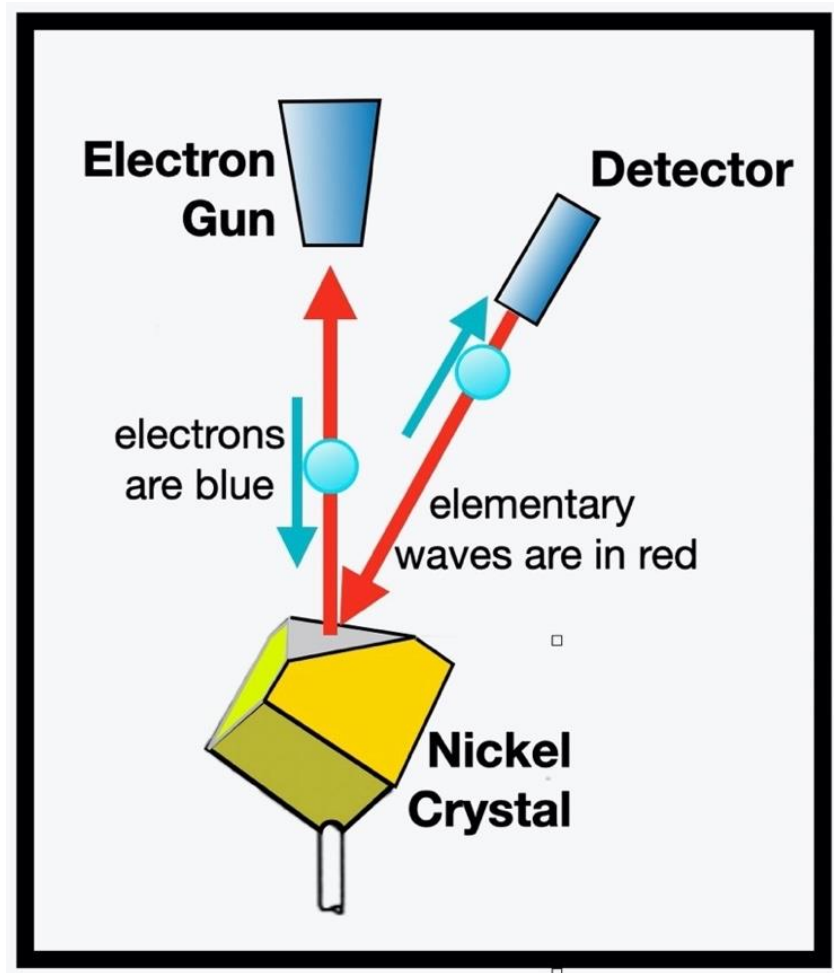

Fig. 11. An elementary wave interpretation of the Davisson Germer experimental data: electrons (blue) follow elementary waves (red) backwards.

This experiment also doesn't tell us in which direction the waves are traveling. The Davisson Germer data are consistent with the idea that elementary waves of about $1.67 \AA$ wavelength start at the detector, deflect 
backwards through the crystal and go up to the electron gun, whereupon electrons follow the waves backwards through the crystal and register at the detector (Fig. 11).

\section{Quantum Electrodynamics (QED) - is the $\mathbf{5}^{\text {th }}$ reason discard wave particle duality}

Our theory, TEW, is based on the premise that $-\psi$ is the wavefunction used by nature. If that were true, the Periodic Table would need to be revised because it should be based on negative orbitals, not orbitals. We discussed this astounding idea in a previous article in this journal. It would produce no changes to electron bonding, and therefore no empirical changes to chemistry.(12)

If the difference between nature's $-\psi$ and QM's $+\psi$ is not evident in chemistry, where would it be observable? In free particles! TEW's $-\psi$ requires that waves start at detectors and move to the particle source. A free particle then chooses which of the impinging waves to follow backwards. We demonstrated previously that TEW can explain Feynman's QED (Quantum ElectroDynamics).(13) His "propagator" (kernel) $+K$ becomes a "reverse propagator" $-K_{R} \cdot(13,46)$ Feynman's pathways are bi-directional, like paths in the woods that can be hiked in either direction (Fig. 12). We propose that a reverse propagator integrates across the path from detector to particle source, then the particle randomly selects only one path to follow back to the detector.

A rule of mathematics is that reversing the bounds of integration causes an integral to become its negative.

$$
\int_{b}^{a} f(x) d x=-\int_{a}^{b} f(x) d x \quad \forall f
$$

As we said before, this does not involve time reversal.

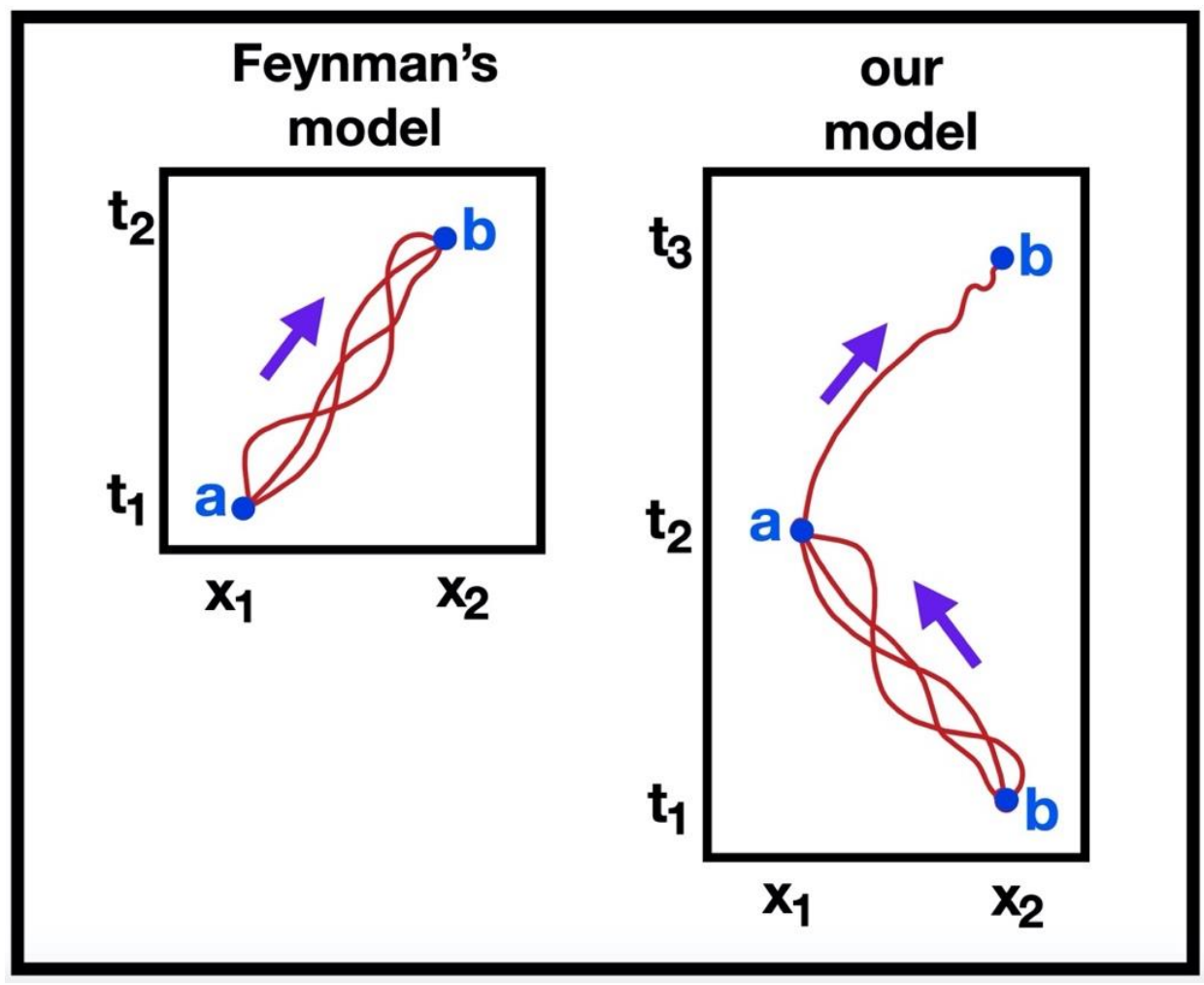

Fig. 12. The lower half of the right diagram postulates that before a free particle is emitted the reverse propagator $-K_{R}$ (an aggregate of all paths) integrates from detector $(\mathbf{b})$ to particle source $(\mathbf{a})$. The particle then selects just one path to follow from (a) to (b) (right top). With TEW the stopwatch starts before a particle is emitted. This means that in some experiments, such as the Bell-test-experiments, TEW can obey the lightspeed limit, yet appear to violate that limit, because the TEW stopwatch started before particle emission.

Feynman defines his propagator $(K)$ by integrating from the particle source to the detector. We define our reverse propagator $\left(K_{R}\right)$ by integrating in the opposite direction. Therefore, integration of the reverse propagator 
is the negative of Feynman's path integral: $-K_{R}=+K$. Wave function collapse occurs as a particle is emitted (Figs. $12,14-15)$. As a particle is emitted from (a) it randomly chooses which path to follow to (b).

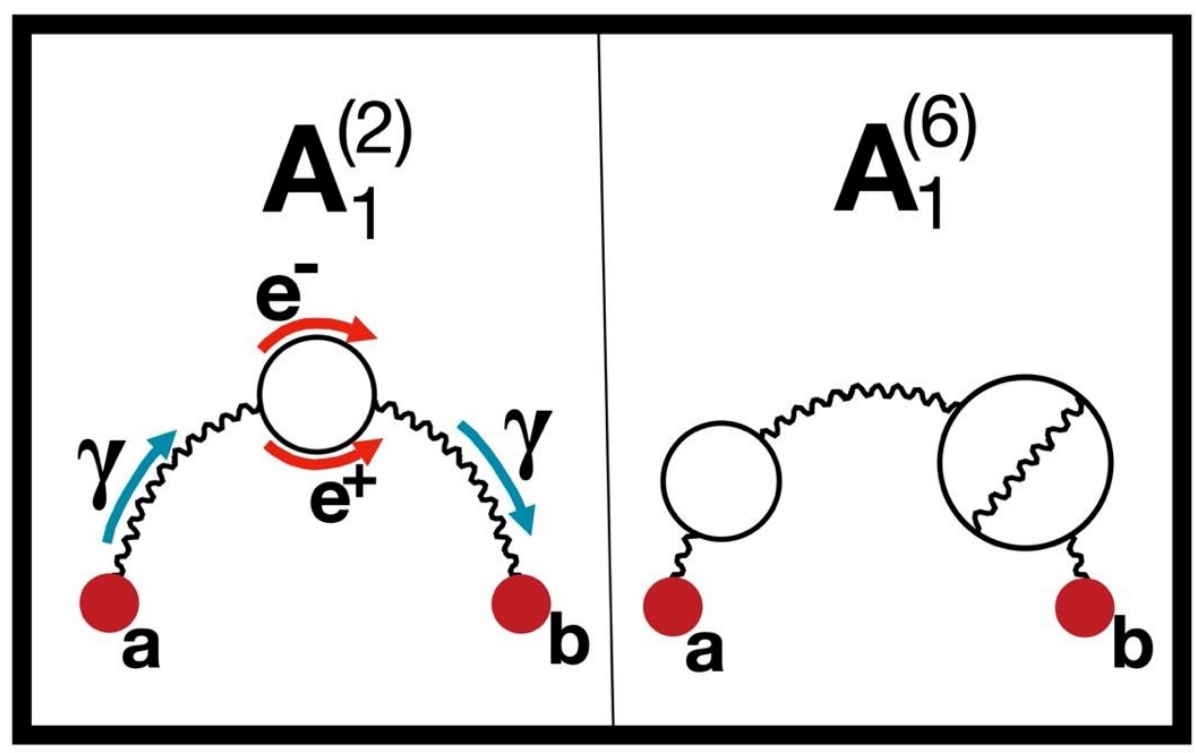

Fig. 13. These are two of the Feynman diagrams used in the Gabrielse study. There are two branch points in the left diagram which makes it a second order Feynman diagram. The right diagram has six. Gabrielse's report includes 891 Feynman diagrams with eight branch points.(47)

If you use Feynman diagrams (Fig. 13), the more diagrams you include, the more accurate are your calculations.(47) Why? Feynman said it was because every particle always follows every pathway, and there are an infinite number of pathways. His students said that made absolutely no sense. They said one particle can only use one Feynman diagram. We agree with his students.

Feynman had it backwards. First waves traverse every Feynman diagram from detector (b) to particle source (a). Then a particle chooses only one Feynman diagram to travel from (a) to (b). The probability of going from (a) to (b) equals the absolute square of integrating the reverse propagator from (b) to (a):

$P=\left|-K_{\mathrm{R}}\right|^{2}=|+K|^{2}$. As you recall, we re-interpret Max Born's rule as $P=|-\psi|^{2}$ rather than $P=|+\psi|^{2}$. Now we extend that viewpoint to Feynman's propagator, interpreting Born's rule as $P=\left|-K_{R}\right|^{2}$ rather than $P=|+K|^{2}$.

Comparing our idea to Feynman's: both explain why the more Feynman diagrams you include, the more accurate your calculations. Common sense tells us that one particle takes only one pathway, not 891 pathways. Do you believe that every photon from this page travels by every possible pathway to reach your retina? Surely you believe that each solitary photon takes only one path up into your retina! Feynman's explanation bulldozes common sense. It asks you to ignore contradictions and forget common sense. TEW makes sense of Feynman diagrams, because it explains why "the more Feynman diagrams you include, the more accurate your calculations" yet TEW agrees with Feynman's students that one particle can only take one pathway.

\section{Defining "Wave Function Collapse"}

So far, our reasoning has been consistent with the thesis that wave function collapse occurs when a particle is emitted, not when it is observed. But is that thesis correct? No!

Sometimes a free particle acquires its final characteristics after it is emitted but before it is observed. For example, two electrons approaching each other at relativistic speeds veer away to avoid one another. Because of the Pauli exclusion principle, they affect each other's polarity. If one electron is spin up, the other becomes spin down.(45) 


\section{The meaning of "wave function collapse"}

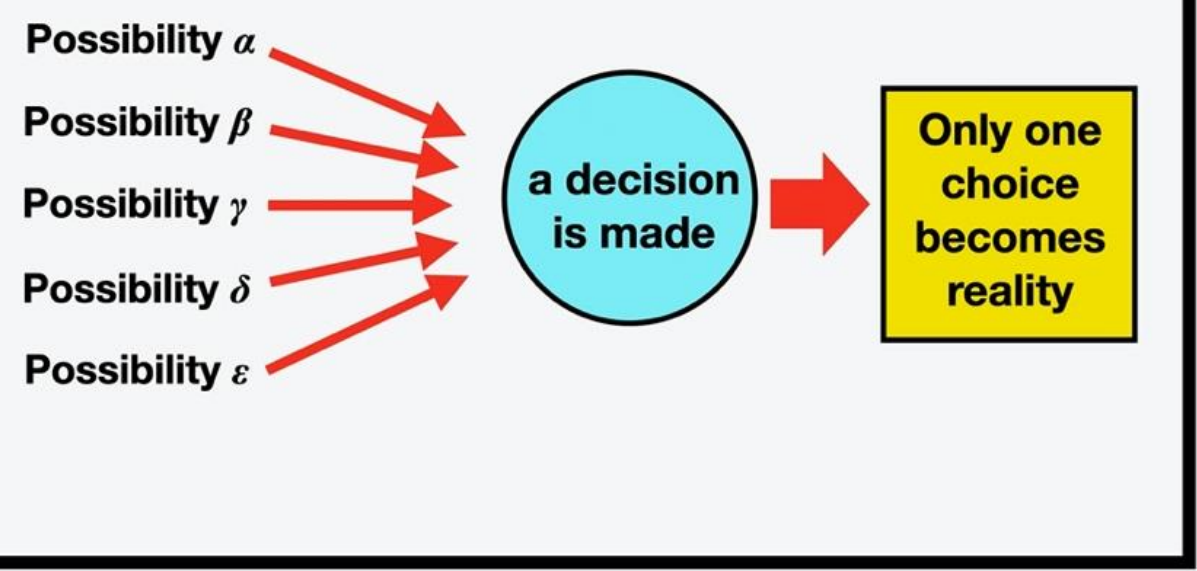

Fig. 14. This defines "wave-function-collapse." It means a decision is made.

We propose that wave function collapse occurs before a particle is observed but is not necessarily located at the particle gun when it is emitted.

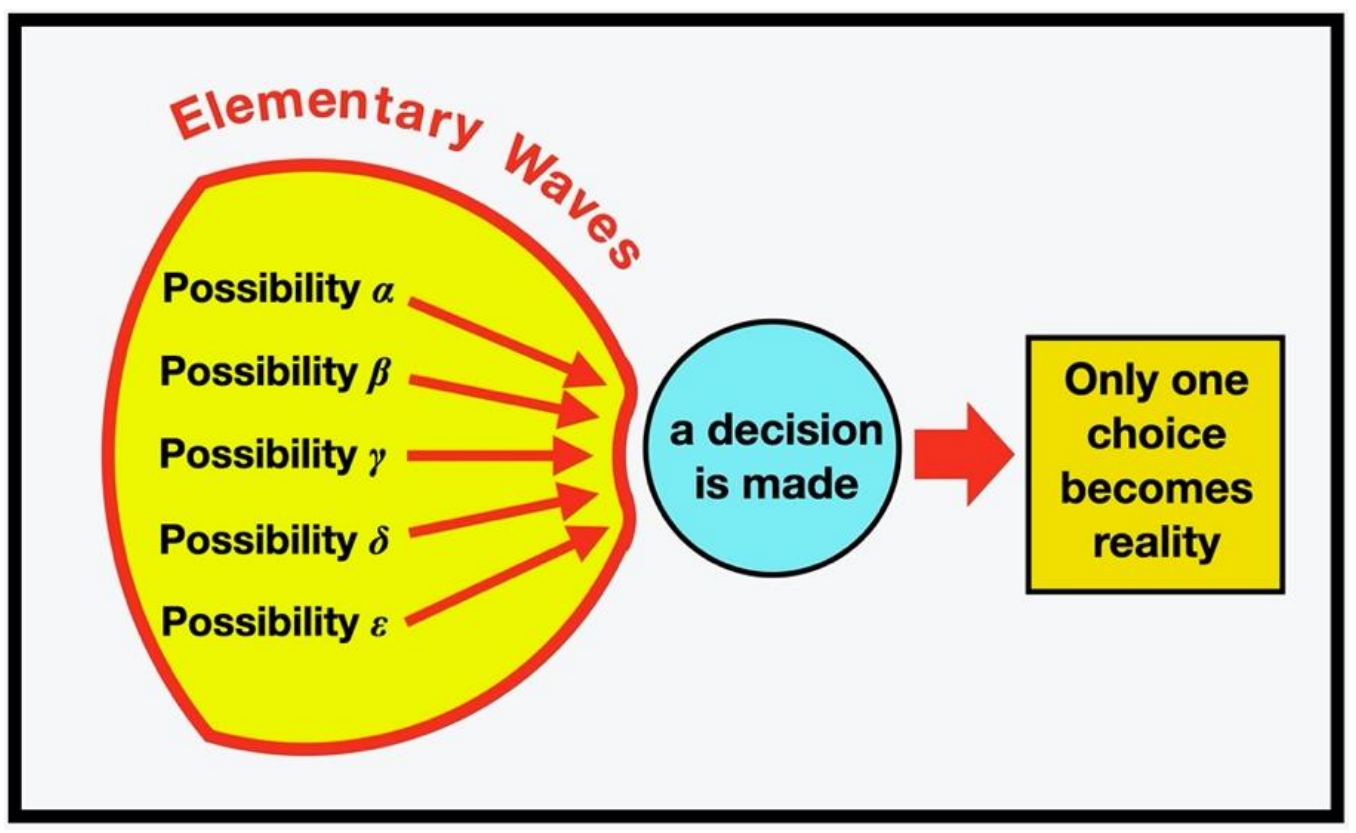

Fig. 15. This summarizes TEW. "Possibilities" means "elementary waves." What we call "a-decision-is-made" others call "wave-function-collapse."

Figs. 12, 14 and 15 define "wave function collapse," in a way that is relevant to the Feynman path integral model. Feynman would say that wave-function-collapse occurs when a particle is observed or measured, whereas TEW says that wave-function-collapse occurs when a particle is emitted in response to one randomly selected elementary wave.

\section{Bell Test Experiments}

We come now to the sixth of six reasons to discard wave particle duality. We will discuss the Bell test experiments. Although Einstein's local realism has been discarded based on loophole free Bell test experiments, there is no 
consensus concerning what should replace Einstein's local realism. TEW offers a choice that is not Einstein's local realism, nor is it QM. $(1-3,6-9,17,19-21,24,32,36,41,49-54,57-58,60,64-65,67-68,70)$

Other people say that nonlocality occurs due to the phenomenon of entanglement, whereby particles that interact with each other become permanently correlated, or dependent on each other's states and properties, to the extent that they effectively lose their individuality and, in many ways behave as a single entity. But everything is chaotic in the intellectual world. For example, some people say that things that are going on here and now are immediately affected by things that are happening on the other side of the galaxy, 106 thousand light years away. If you are absent-minded, it would be absurd to blame that flaw on the entanglement of your brain molecules with molecules on the other side of the Milky Way galaxy.

Let's discuss the term "nonlocal" further. For a century after Isaac Newton, people said that gravity was "nonlocal." The sun magically reached across empty space and interacted gravitationally with each planet. This "nonlocality" troubled Newton, who thought it was a weakness in his theory. Subsequently science developed the idea of a gradient or field. Each position in space was locally affected by a field.(48). Similarly, it was previously thought that Alice's equipment magically reached across empty space and sent a signal to Bob's equipment. TEW solves that problem of the distance between Alice and Bob, without exceeding lightspeed. This is accomplished when TEW defines a field all the way from Alice, through the 2-photon source, and to Bob. Everywhere in that field the coincidence rate is $\cos ^{2}\left(\varphi_{2}-\varphi_{1}\right)$, as you will see. There is no gradient, simply an omnipresent $\mathrm{P}=\cos ^{2}\left(\varphi_{2}-\varphi_{1}\right)$ throughout the fibreoptic cable.

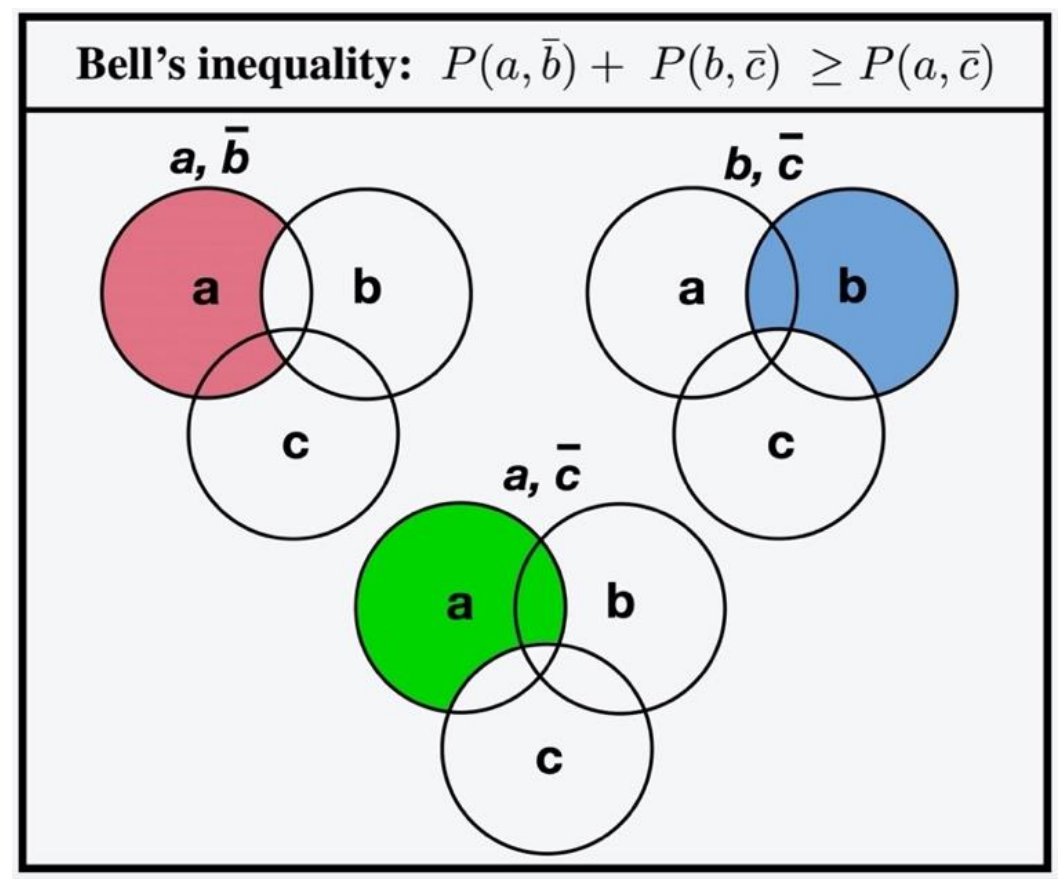

Fig. 16. Venn diagrams of Bell's inequality, describing local realism. The letters " $a, \bar{b}$ " mean "yes a, not b" which refers to the pink area that includes all the area of circle "a" that is not included in circle "b". Similarly for the letters " $b, \bar{c}$ " for the blue circle and " $a, \bar{c}$ " for the green circle. 


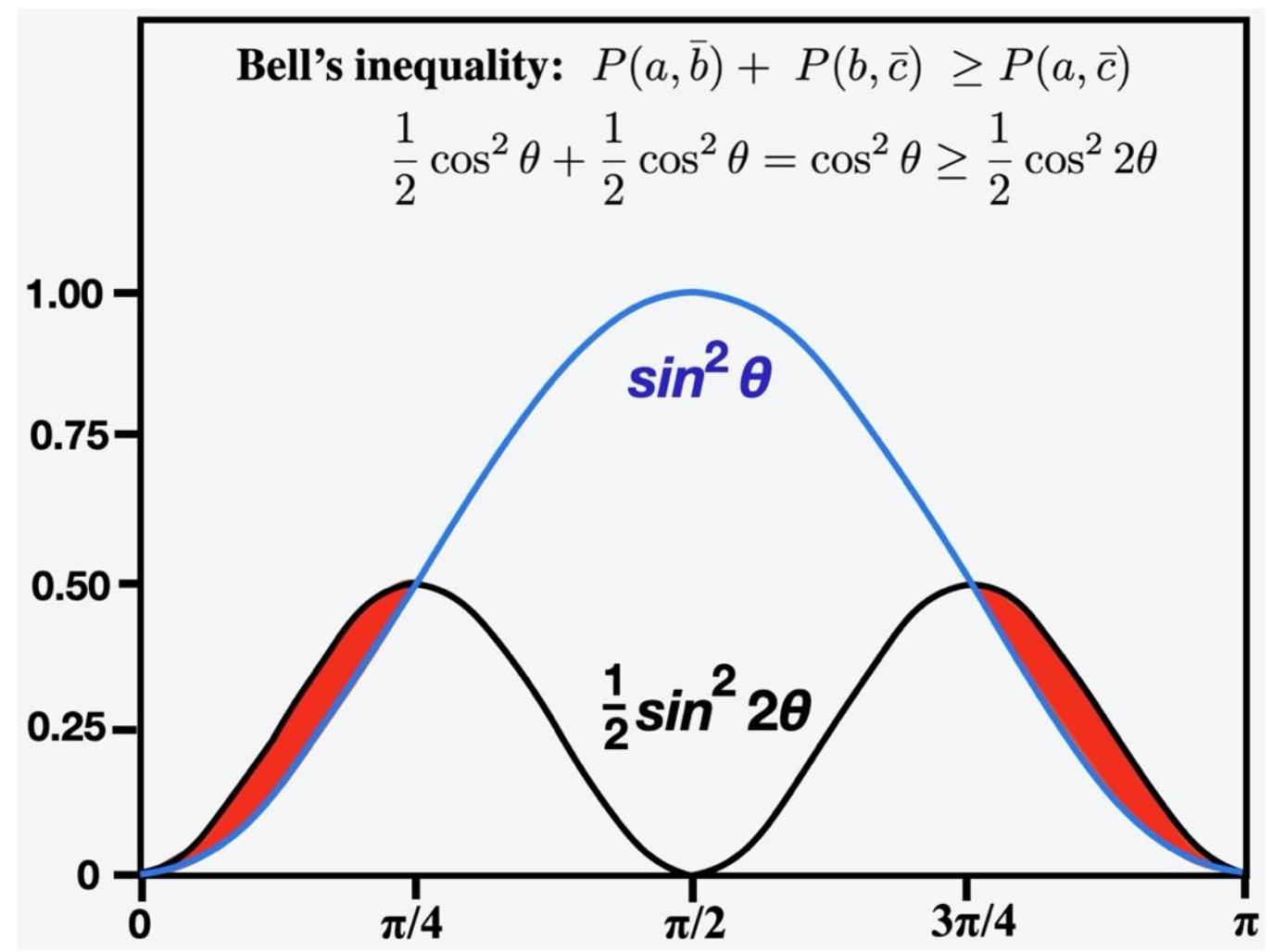

Fig. 17. Bell's inequality applied to experiments in which Alice and Bob each use a polarizer set at a random angle and calculate the coincidence rate at which they both detect a photon. $\theta$ is the difference between the angle at which their polarizers are set. Bell's inequality is violated in the red areas, which cannot be explained by Einstein's local realism.

In order to analyze the Bell test experiments we need a more sophisticated understanding of TEW. The assumption of TEW is that at every point of space there are an infinite number of zero-energy elementary waves travelling at the speed of light in all directions and all wavelengths. This implies that every elementary ray has a mate, namely an elementary ray travelling coaxially in the opposite direction. We use the symbol $\boldsymbol{A}$ for an elementary ray, and $\boldsymbol{A E} \rightleftarrows \boldsymbol{A}$ for a Bi-Ray (see Fig. 20). What makes the two rays coherent is the photon(s) following that Bi-Ray.

John Bell defined the inequality equation shown in Fig. 16. His equation and these Venn diagrams describe our everyday experience. The astonishing thing is that this logic is contradicted by Bell test experiments.

Experiments using entangled particles have rejected Bell's inequality, which means that either locality or reality is wrong. $(49-52,58,64)$ In Fig. 17 theta $(\theta)$ is defined as the angle between Alice and Bob's polarizer angles $\left(\varphi_{1}\right.$ and $\varphi_{2}$ respectively). Many experiments have demonstrated that the top blue curve in Fig. 17 (i.e. coincidence rate $=\sin ^{2} \theta$ ) is true of nature, and rejects the Bell inequality in the red areas.

Although the coincidence rate of $\sin ^{2} \theta$ defines test results that violate Bell's inequality, if we rotate the axes, the coincidence rate of $\cos ^{2} \theta$ tells us the same thing. One experiment found a coincidence rate of $\sin ^{2}(\theta+x)$, where the variable " $x$ " varied depending on the time of the day, as the temperature of the equipment changed.(57,65,67) That entire family of sinusoidal squared curves violates Bell's inequality. Alain Aspect et.al. reported a coincidence rate of $\cos ^{2} \theta$ in 1982.(2)

How does TEW arrive at the prediction that the coincidence rate is $\cos ^{2}(\theta)$ ? Consider the apparatus used by Alain Aspect (Fig. 18). Entangled photons from a calcium cascade Source (S) travelled in opposite directions and were either detected or not detected by equipment denoted as "Alice" and "Bob." While the photons were in flight, the devices named $C_{1}$ and $C_{2}$ randomly rotated the angle at which the polarizers were set. The devices named $C_{1}$ and $C_{2}$ consisted of small pools of water with waves vibrating across the surface in an unpredictable pattern that was considered to be random. 


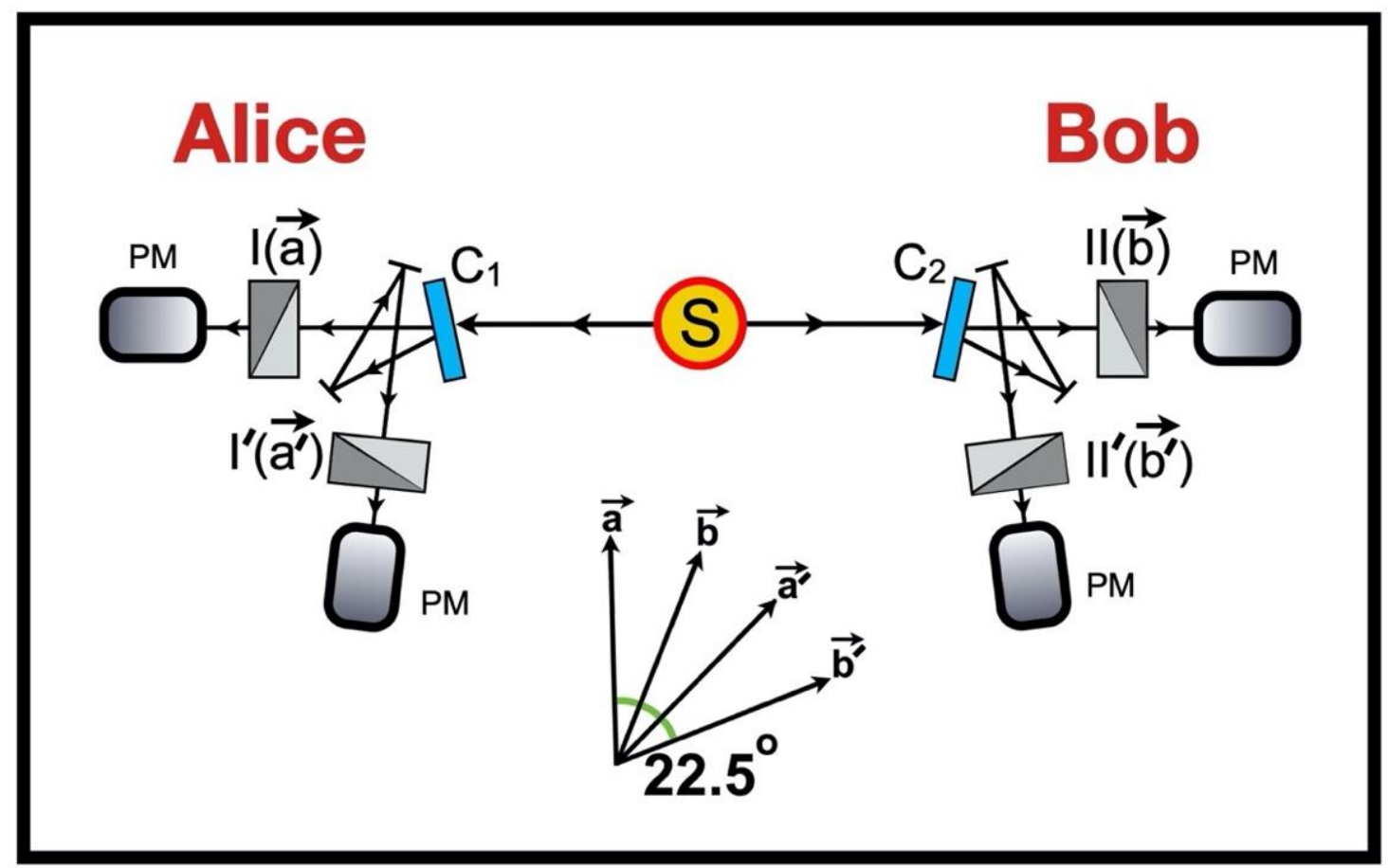

Fig. 18. Equipment used by Aspect, Grangier and Roger in 1982. Entangled photons departed the source "S" and were assigned by a randomizing device $\left(C_{1}\right.$ or $C_{2}$ shown in blue) to Wollaston prisms angled at $22.5^{\circ}$ increments, and then observed by photomultipliers (PM). The angles of measurement were chosen to maximize the discrepancies between QM and Einstein's predictions.

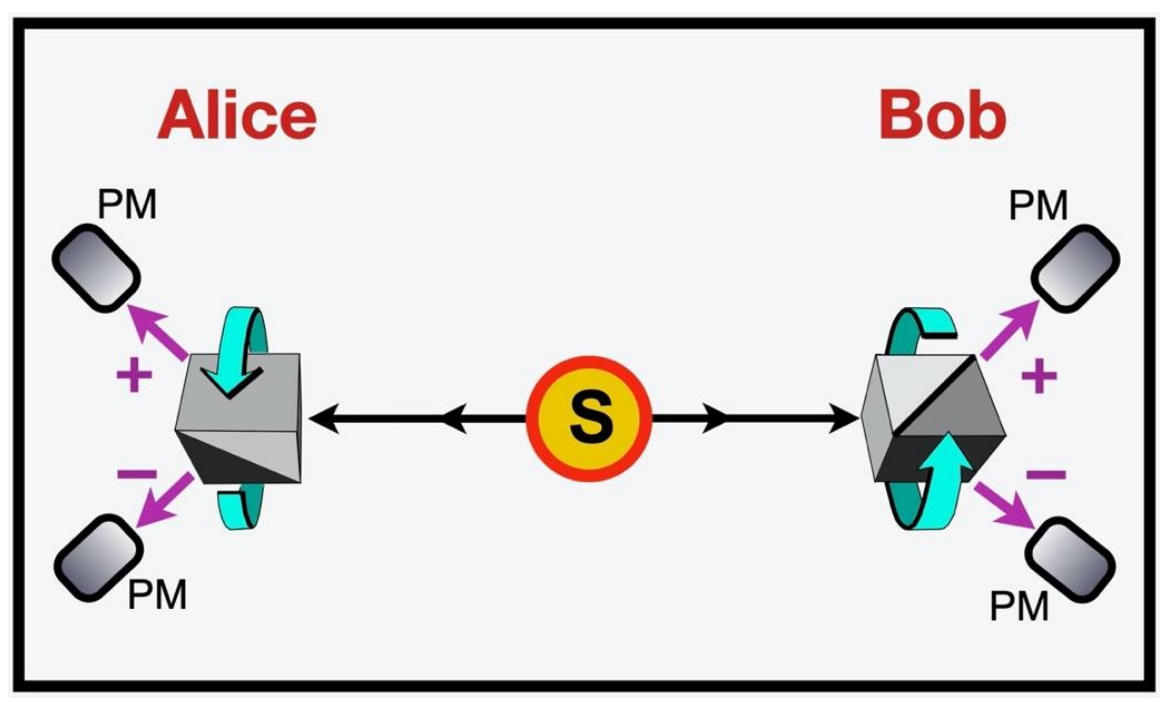

Fig. 19. The apparatus shown in Fig. 18 can be generalized to use continuous variables as the angle between the polarizers. In this diagram the cubes are Wollaston prisms which can be rotated at random, taking the photomultipliers with them. The randomization occurs after the photons have left the source and are in flight. The results are therefore called "delayed choice."

If a pair of entangled photons start in the center, halfway between Alice and Bob, and both photons follow the same Bi-Ray in opposite directions (Fig. 20). We define the probability of one photon following a Bi-Ray as the amplitude of it following one ray times the amplitude of it following the countervailing ray. That is the only assumption we need in order to explain the Bell test experiments. The only assumption! 


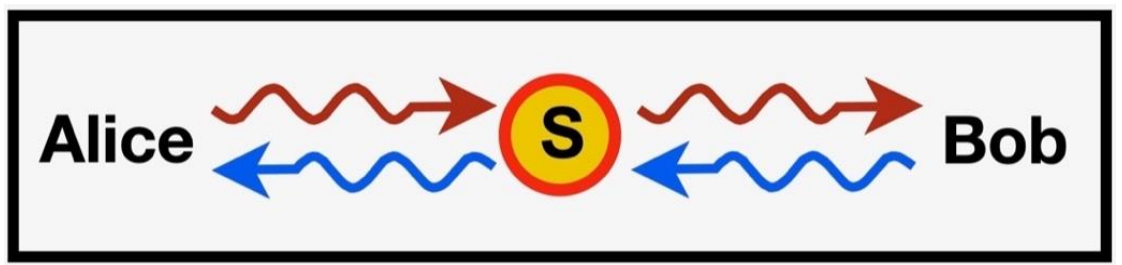

Fig. 20. An elementary ray (in red) from Alice travels to the photon source, passes through that source and reaches Bob's equipment. Similarly, an elementary ray from Bob (in blue) penetrates the Source and reaches Alice. These two rays form a Bi-Ray $(\boldsymbol{A E} \rightleftarrows \boldsymbol{E})$, somewhat like the two rails of a railroad track. Entangled photons are like two locomotives travelling in opposite directions on the same railroad track. All the energy comes from the locomotives, none from the track. The Bi-Ray is established long before photons are emitted.

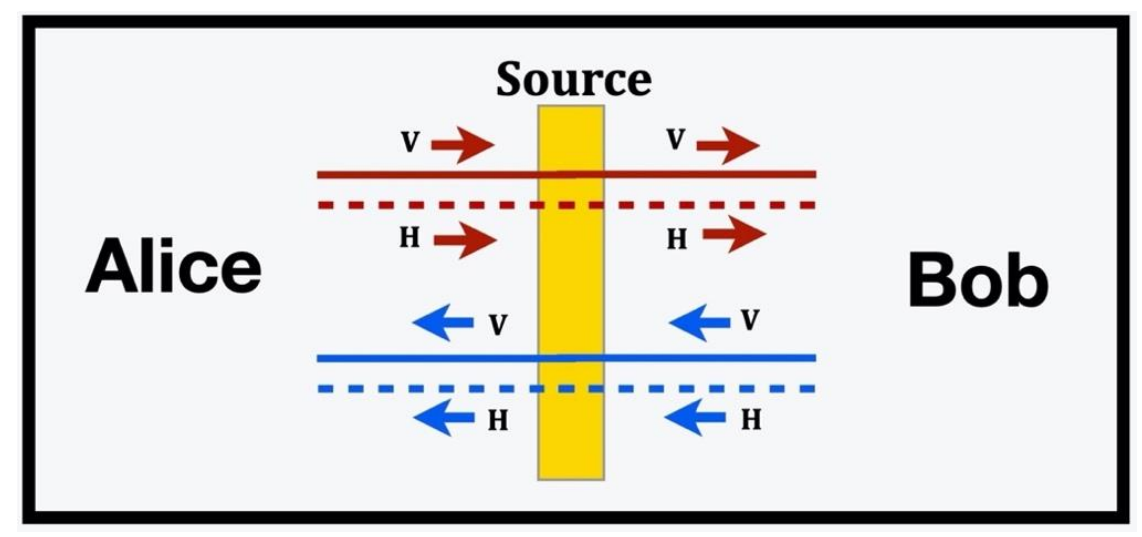

Fig. 21. Using vertical and horizontal eigenstates, we re-draw Fig. 19. We will use red to denote an elementary ray $(\boldsymbol{E})$ travelling to the right, and blue for an $\boldsymbol{A}$ travelling to the left. Note however that these eigenstates apply only to the individual elementary rays (red or blue) but not to the Bi-Ray( $(\boldsymbol{E} \rightleftarrows \boldsymbol{A E})$. In the Figure 22 the four $\mathrm{Bi}$ Ray eigenstates are given.

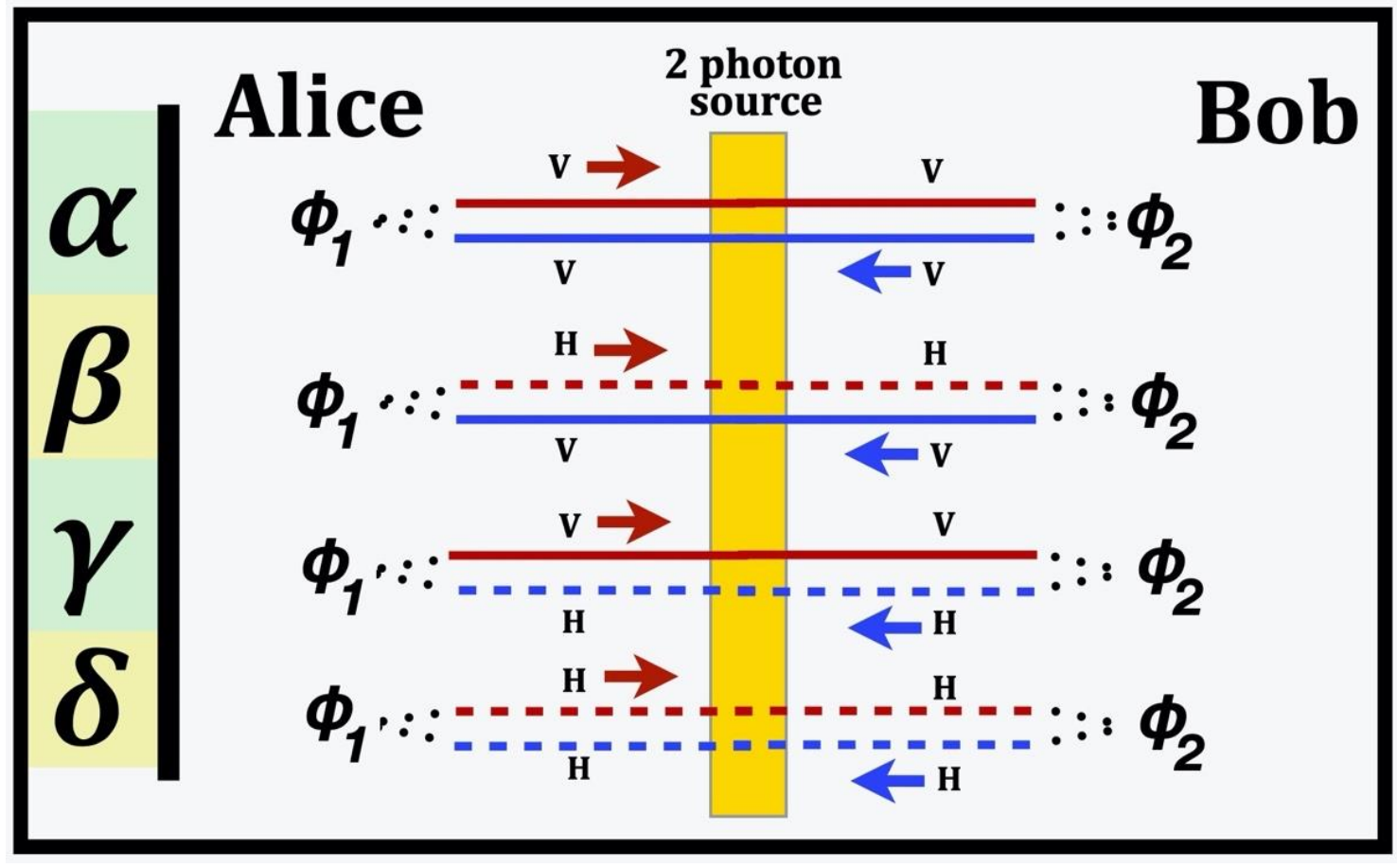

Fig. 22. We define four new eigenstates $(\boldsymbol{\alpha}, \boldsymbol{\beta}, \boldsymbol{\gamma}$ and $\boldsymbol{\delta})$ of the Bi-Ray stretching between Alice and Bob. The angles $\varphi_{1}$ and $\varphi_{2}$ are the random angles of Alice and Bob's polarizers. Neither Alice nor Bob influence or even know what angle of polarization the other person is using. 


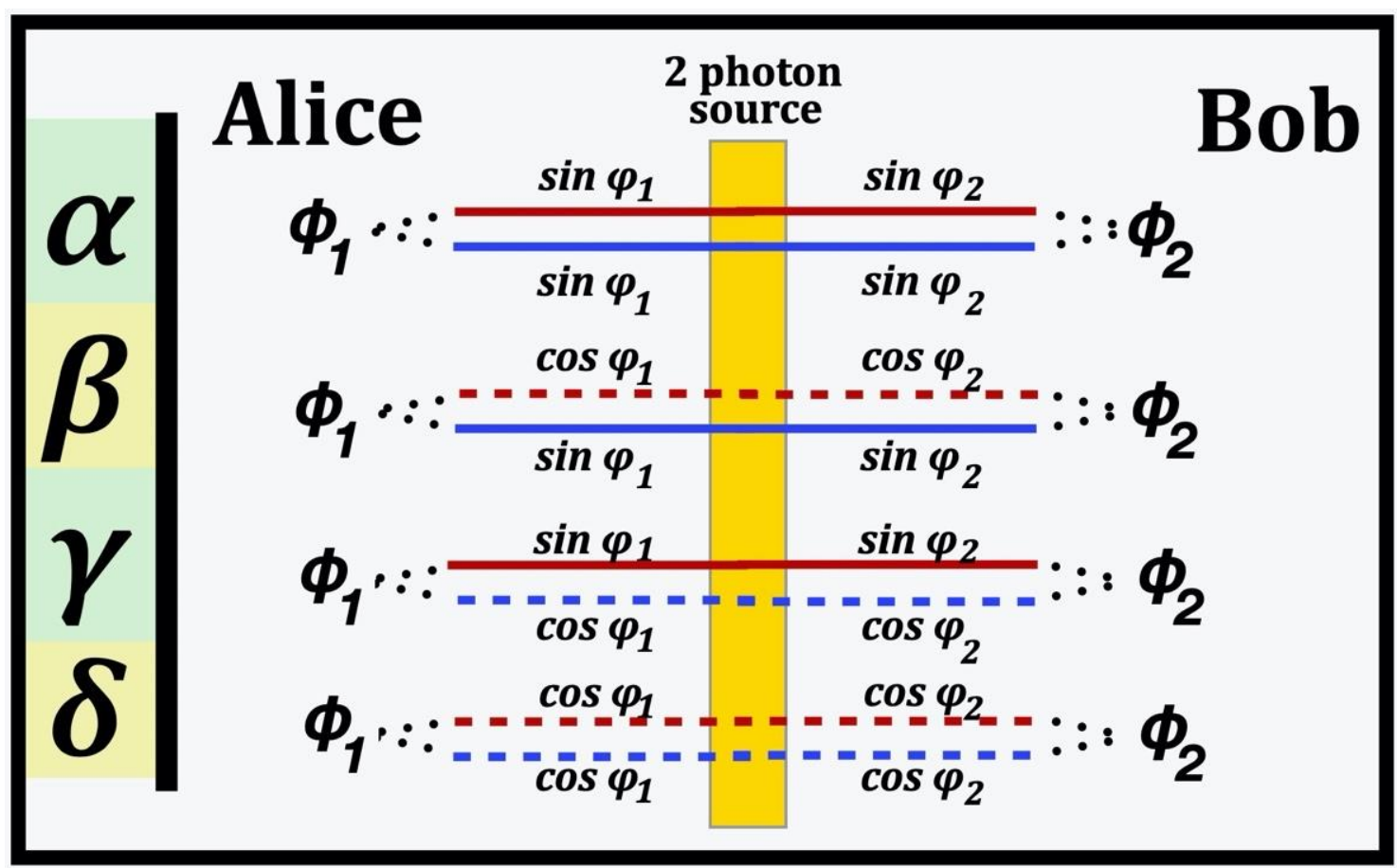

Fig. 23. These sines and cosines show the amplitude for a photon from the source being detected by Alice or Bob in a specific eigenstate, when their polarizers have been set to angles $\varphi_{1}$ and $\varphi_{2}$ respectively. Note that one photon travels to the left and the other photon to the right. Alice's choice of angle $\varphi_{1}$ is independent of Bob's choice of angle $\varphi_{2}$.

Probability of both Alice and Bob simultaneously seeing a photon (the so-called "coincidence rate") in the $\boldsymbol{\alpha}$ eigenstate is the probability of Alice seeing a photon $\left(\sin \left(\varphi_{1}\right) \sin \left(\varphi_{1}\right)\right)$ times the probability of Bob seeing a photon $\left(\sin \left(\varphi_{2}\right) \sin \left(\varphi_{2}\right)\right)$ in that eigenstate. This becomes the first line of the equation

$$
\mathbf{P}=\sin \left(\varphi_{1}\right) \sin \left(\varphi_{1}\right) \times \sin \left(\varphi_{2}\right) \sin \left(\varphi_{2}\right) \ldots
$$

To find the probability of Alice and Bob simultaneously seeing a photon (called the "coincidence rate"), we add together the four eigenstates $(\boldsymbol{\alpha}, \boldsymbol{\beta}, \boldsymbol{\gamma}$ and $\boldsymbol{\delta})$ in Fig. 23:

$\mathbf{P}=\sin \left(\varphi_{1}\right) \sin \left(\varphi_{1}\right) \times \sin \left(\varphi_{2}\right) \sin \left(\varphi_{2}\right) \leftarrow($ within eigenstate $\alpha)$

$+\cos \left(\varphi_{1}\right) \sin \left(\varphi_{1}\right) \times \cos \left(\varphi_{2}\right) \sin \left(\varphi_{2}\right) \leftarrow($ within eigenstate $\beta)$

$+\sin \left(\varphi_{1}\right) \cos \left(\varphi_{1}\right) \times \sin \left(\varphi_{2}\right) \cos \left(\varphi_{2}\right) \leftarrow($ within eigenstate $\boldsymbol{\gamma})$

$+\cos \left(\varphi_{1}\right) \cos \left(\varphi_{1}\right) \times \cos \left(\varphi_{2}\right) \cos \left(\varphi_{2}\right) \leftarrow($ within eigenstate $\delta)$

When we add those four lines together, the result can be factored:

$=\left[\sin \left(\varphi_{1}\right) \sin \left(\varphi_{2}\right)+\cos \left(\varphi_{1}\right) \cos \left(\varphi_{2}\right)\right]$

$x\left[\sin \left(\varphi_{1}\right) \sin \left(\varphi_{2}\right)+\cos \left(\varphi_{1}\right) \cos \left(\varphi_{2}\right)\right]$

And there is a trigonometry relationship that allows us to compress those two lines into two functions:

$=\cos \left(\varphi_{2}-\varphi_{1}\right) \times \cos \left(\varphi_{2}-\varphi_{1}\right)$

$=$ "coincidence rate" $=\cos ^{2}\left(\boldsymbol{\varphi}_{2}-\boldsymbol{\varphi}_{1}\right)$ 
This is how TEW accounts for the Bell test data. The math is astonishingly simple. Our only starting assumption

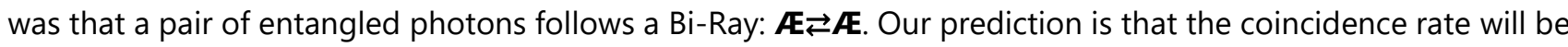
$\mathbf{P}=\cos ^{2}\left(\boldsymbol{\varphi}_{2}-\boldsymbol{\varphi}_{1}\right)$. If the 2-photon-Source were changed so it emitted photons orthogonal to one another, then the final coincidence rate would be $\mathbf{P}=\sin ^{2}\left(\varphi_{2}-\varphi_{1}\right)$.

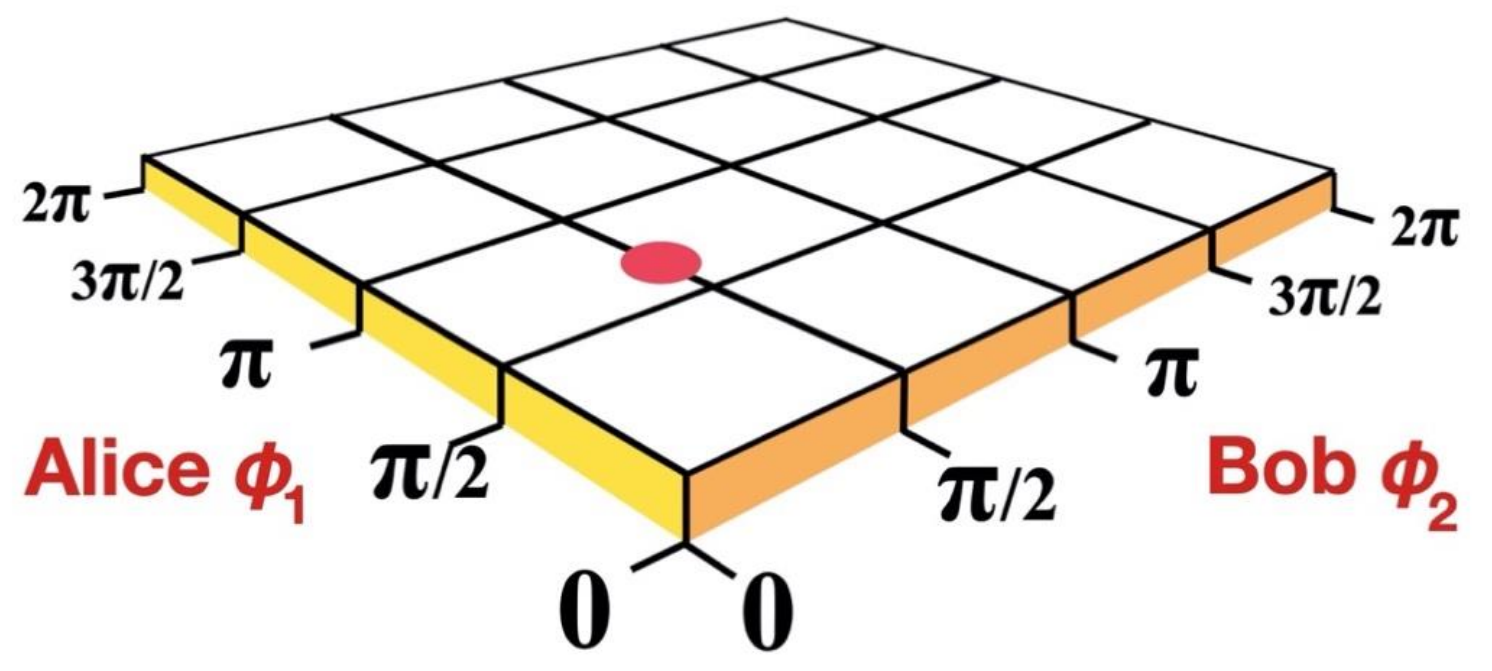

Fig. 24. This is a graph of every possible setting of Alice's and Bob's polarizers. For example, the red dot would correspond to Alice's $\varphi_{1}=2 \pi / 3$ and Bob's $\varphi_{2}=\pi / 2$. In the next diagram we add the " $Z$ " axis.

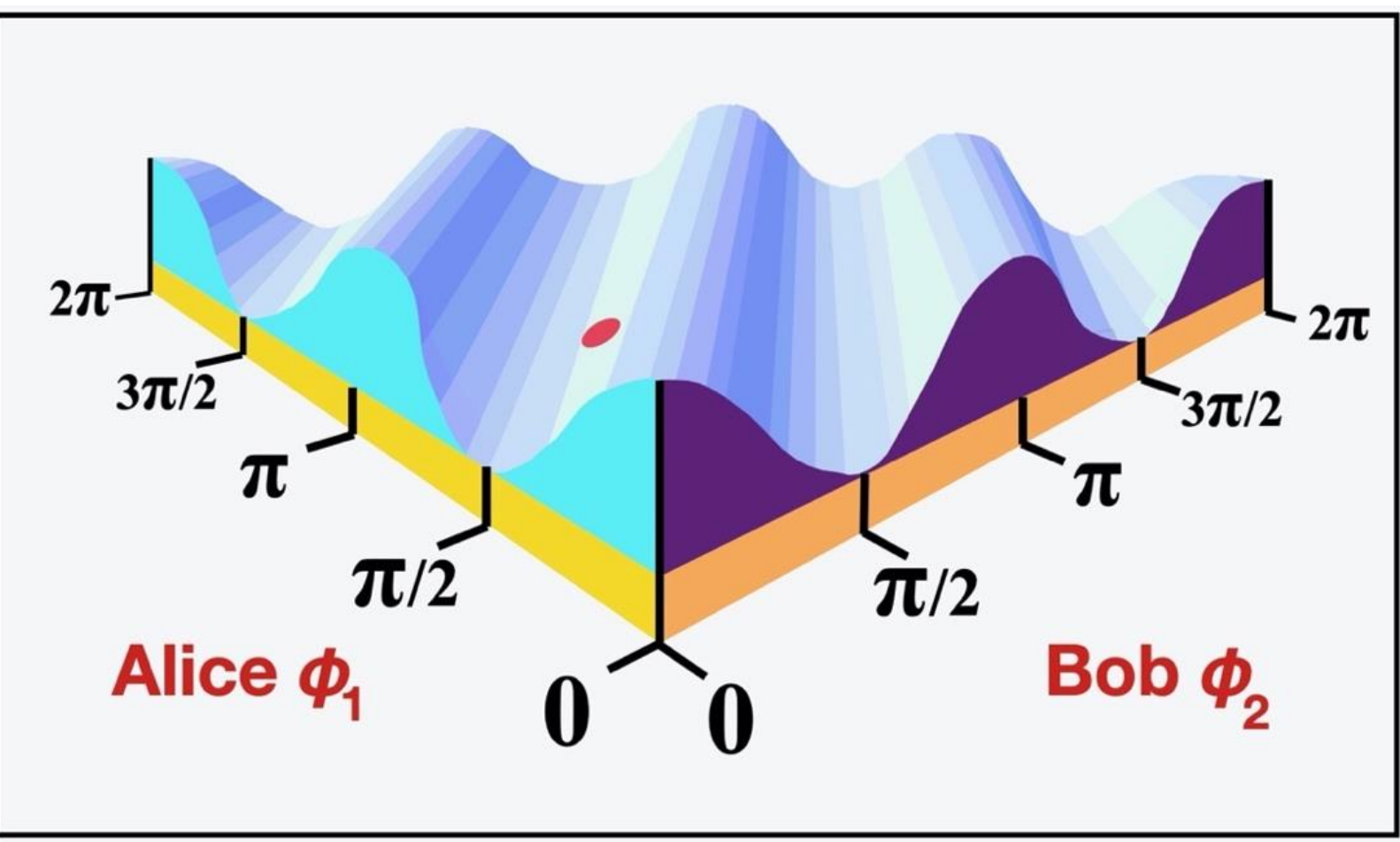

Fig. 25. This is a graph of $z=\cos ^{2}\left(\varphi_{2}-\varphi_{1}\right)$. The "Z" axis (i.e. the depth of what appears to be ocean waves) shows the coincidence rate $(P)$ for them both seeing a photon simultaneously. Alice is free to choose any angle $\varphi_{1}$ and Bob any angle $\varphi_{2}$. Neither of them knows what angle the other one chose. 
TEW achieves the unthinkable: it allows quantum math to stretch across space without exceeding the lightspeed limit. TEW obeys the speed-of-light rules but starts earlier than photon emission and therefore appears to violate the future light cone rules. By the time a photon pair is emitted, Bi-Rays are long since established. Wave function collapse (consisting of each entangled photon attaching to the same Bi-Ray) occurs as the photons are emitted and not when the photons are measured by Alice and Bob's detectors.

Our approach rejects the local realism of EPR. Our starting assumptions differ from those of QM. Therefore, the TEW model is both non-Einstein and non-QM. But we are closer to QM than to Einstein.

Reality is different than Einstein thought. Things like entangled photons don't have intrinsic and fixed properties. Instead, they can enter into relationships (a synonym for "elementary waves"), each of which offers a different possibility, as shown in Fig. 25.

What is the "decision" in the Bell test experiments? It is the choice of polarizer angles by Alice and Bob. The probability that they both see a photon simultaneously is $\mathrm{P}=\cos ^{2}\left(\varphi_{2}-\varphi_{1}\right)$ which we take to be a synonym for "coincidence rate".

Einstein thought each particle had solid characteristics (hidden variables) even before it was observed. TEW says that is wrong. It is not the intrinsic characteristics of particles, but the elementary waves that constitute the canvas upon which reality is painted. The characteristics of each particle depend on which angle it is observed at. Each person who asks a different question, will get a different answer (Fig. 25).

Given the battle that raged around Einstein's view, the vocabulary has become charged and misleading. "Local realism" has come to mean Einstein's view, and "nonlocal" has been reified into the QM view. These words are useless and confusing when trying to describe TEW. Some people say passionately that "TEW is a local theory." Others say passionately "TEW is a nonlocal theory." They both agree on how the TEW model works, but they use divergent definitions of the terms "local" and "nonlocal." The best way to describe TEW is to describe it, as we have done, and avoid efforts to put it into a pigeonhole. The usual vocabulary describing Bell test experiments defines pigeonholes that are obsolete and don't fit the TEW way-of-thinking.

We defined TEW as being the theory based the negative wavefunction $-\psi$, the opposite of the wavefunction of $\mathrm{QM}(+\psi)$. We need to explain how that minus sign is relates to our explanation of the Bell test experiments.

On a practical level there are two differences between $-\psi$ and $+\psi$. One is that the Periodic Table should be based on negative orbitals instead of orbitals. That change would not affect electron bonding, and therefore chemistry would be unchanged. Second, free particles would follow elementary waves backwards: starting at a detector and ending at the particle source. We presented empirical evidence of that in a previous article in the Journal of Advances in Chemistry.(12)

In Bell test experiments you have two free particles moving in opposite directions. If those two particles are entangled, then we employ the mathematics of the Bi-Rays $(\boldsymbol{A} \rightleftarrows \boldsymbol{A})$.

When QM sees two entangled particles in a Bell test experiment performing a choreographed dance, they invoke the doctrine of "nonlocality" because the choreography is consistent with quantum math, but there is no mechanism for that math to reach across empty space. TEW provides a mechanism, at the speed-of-light, but not faster. The elementary Bi-Rays were extending from Alice's equipment to Bob's equipment even before the photons were emitted! But be aware that the future light cones published in Bell test experimental research is wrong, because we say the stopwatch started much earlier than the researchers realize.

\section{An invitation for the reader to conduct new experiments}

Since TEW and QM almost always predict identical experimental outcomes, we need empirical research to prove which theory of nature is accurate. In the research designs below, we need to have a moving part in in order to distinguish them. Since elementary waves move at lightspeed, the moving part only has a few nanoseconds in which to change from one condition to another. 
We give now the design of three experiments for which QM and TEW predict different outcomes. This information comes from an article by us, published in Journal of Advances in Mathematics.(33) These experiments have never been conducted. We invite the reader to design and build these experiments. We have no plans to build them, and no one else does either.

When does interference occur relative to the firing of an electron? TEW says interference occurs prior to or during electron firing; QM says it occurs after electron firing, actually after the electron wave-particle penetrates through the double slit barrier. TEW says there is no interference after an electron is fired; QM says the opposite. Therefore, if we divide time at the point when a single electron is fired, we should be able to design experiments for which the theories predict different outcomes. To conduct these experiments, you need to limit emission to one electron at a time, with a delay for the equipment to be reset before the next electron is fired. Figure 26 shows the first experiment.

The equipment in Figure 26 is designed so both slits are open until that nanosecond when an electron is fired. At that instant a powerful laser fires straight down, blocking the right slit. QM says only one slit is open so therefore there will be no interference fringe pattern on the target screen: just one vertical line (a narrow Gaussian line) behind the left slit. TEW says there will be an interference fringe pattern (as shown in Figure 26) skewed toward the left side of the target screen.

The TEW prediction is based on the idea that wave interference occurs before, or during the electron emission. At that time both slits are open. Therefore, there will be elementary ray interference coming through the slits and interfering as they impinge on the electron gun. That pattern of interference will be encoded as longitudinal waves in the frequency of electron emission. Since the right slit is closed when an electron gets there, only the left slit will be open for electrons to inscribe that pattern on the target screen.

One must be cautious in designing the equipment because a laser is sluggish and tends not to close the righthand slit at that nanosecond when we want the slit closed.

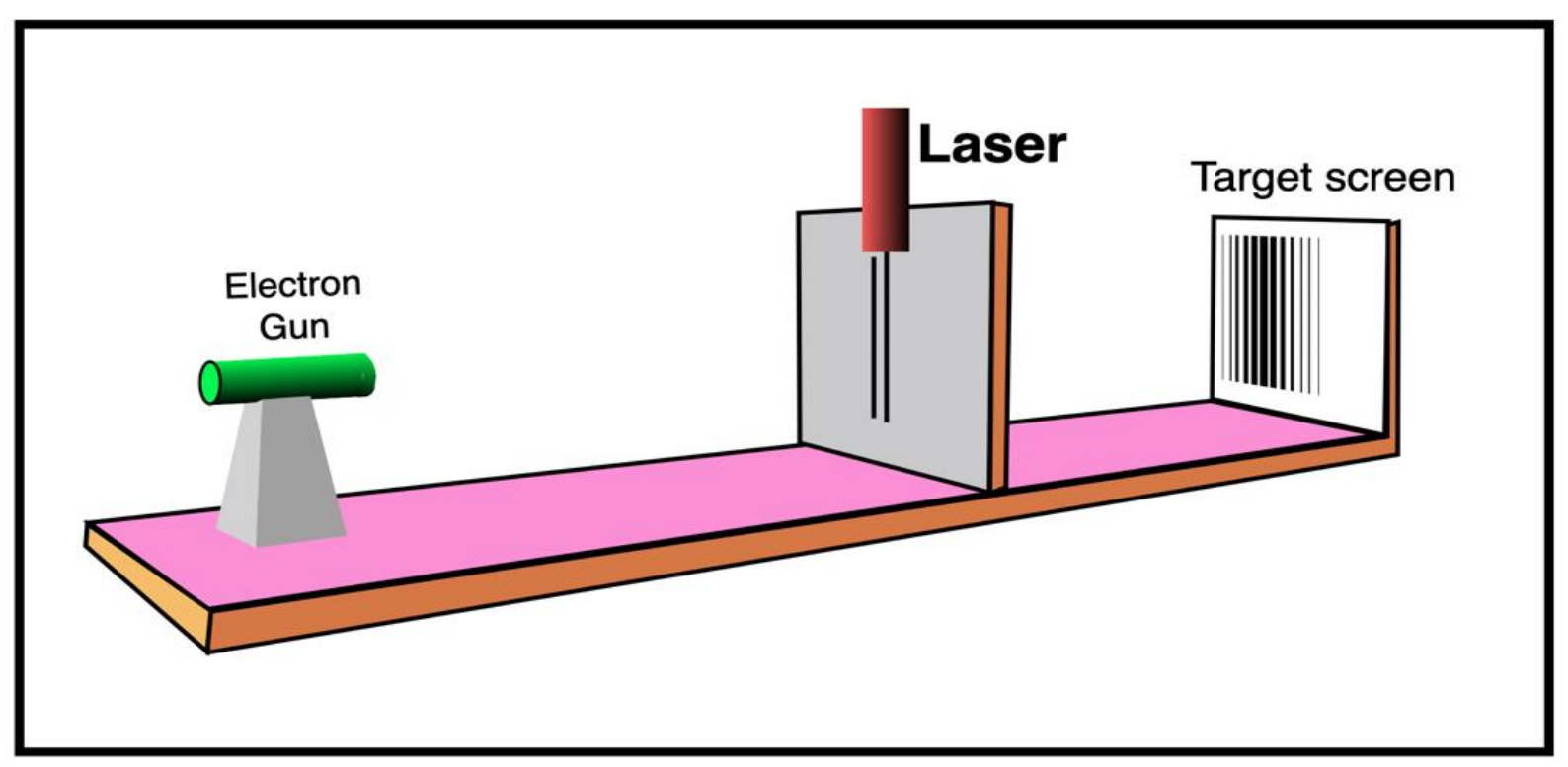

Fig 26. The right slit of a double slit experiment closes at that nanosecond when a particle is fired. The interference wave pattern exists, but is pushed to the left on the target screen.

If this proves to be true, it will refute something Feynman repeatedly said: that it is impossible to both see an interference fringe pattern and know which slit an electron used. One of the axioms of complementarity would be proved wrong.

The next two experiments are based on the apparatus shown in Figs. 27 and 28. This is an experiment learned from Lewis E. Little. This time both slits are always open. A "vanishing screen" is inserted in front of the target screen. The "vanishing screen" needs to be designed so it is opaque to electrons until the nanosecond that an 
electron is fired, and then it becomes transparent. Alternatively, it needs to be transparent to electrons until one is fired, and then it becomes instantly opaque. Electrons are fired one at a time, with a pause in between.

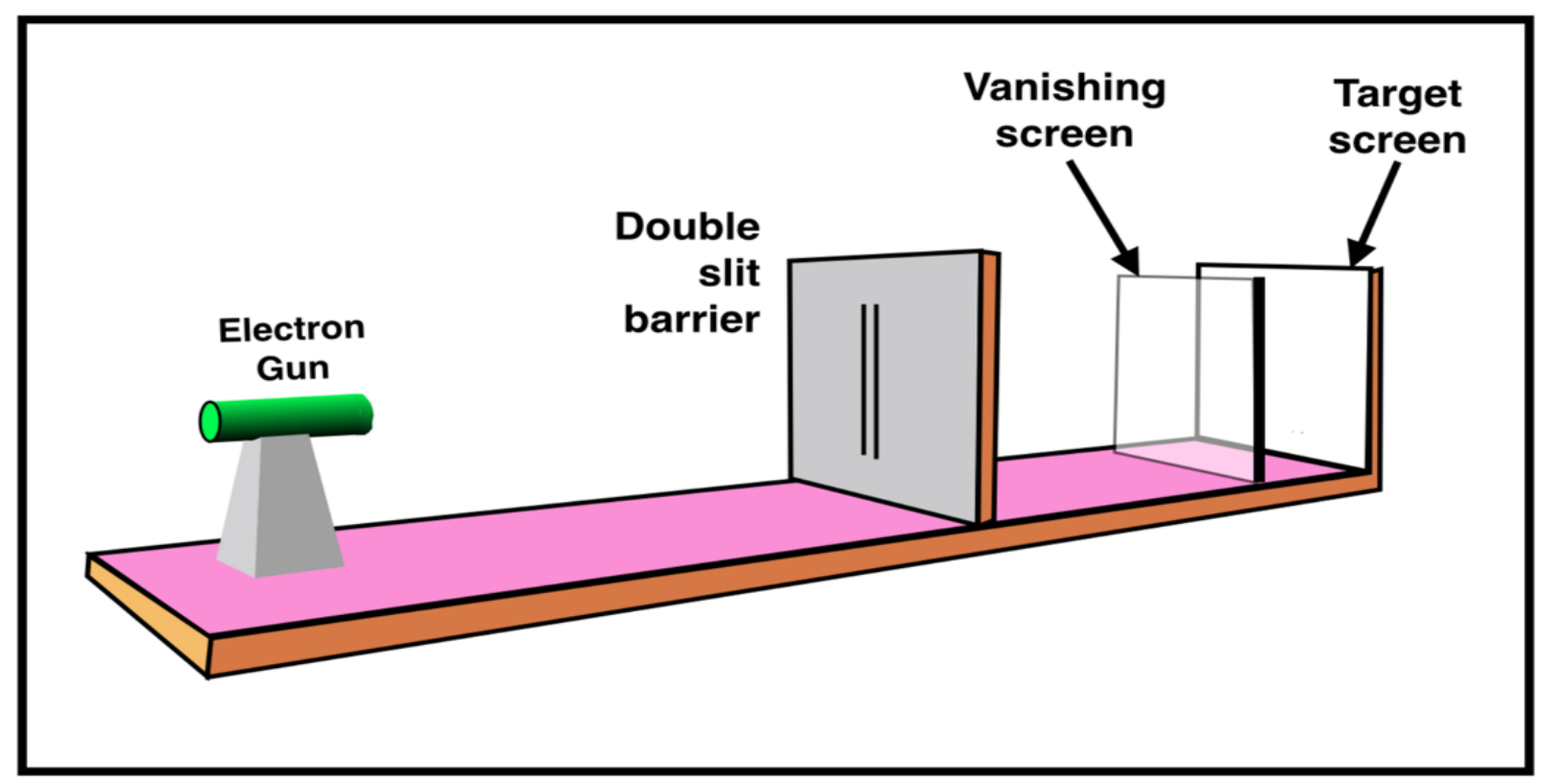

Fig. 27. A screen that switches between opaque and transparent in nanoseconds is inserted into a double slit experiment.

The reason for the unusual predictions by TEW is again because of the timing of elementary wave interference. Starting with an opaque "vanishing screen," elementary rays emanating from all points of that screen will interfere near the electron gun prior to the gun firing. The amplitude of an electron choosing that ray as opposed to the other competitor rays is proportional to the strength of its amplitude. The amplitude reflects the pattern of interference, which we can indirectly see from the blue curve on the home screen (Figure 29). If an electron chooses to follow one elementary ray backwards, it is programmed to strike the home screen (the opaque "vanishing screen") in the familiar aqua-blue pattern. But we tricked nature. The home screen is nowhere to be found, having become transparent.

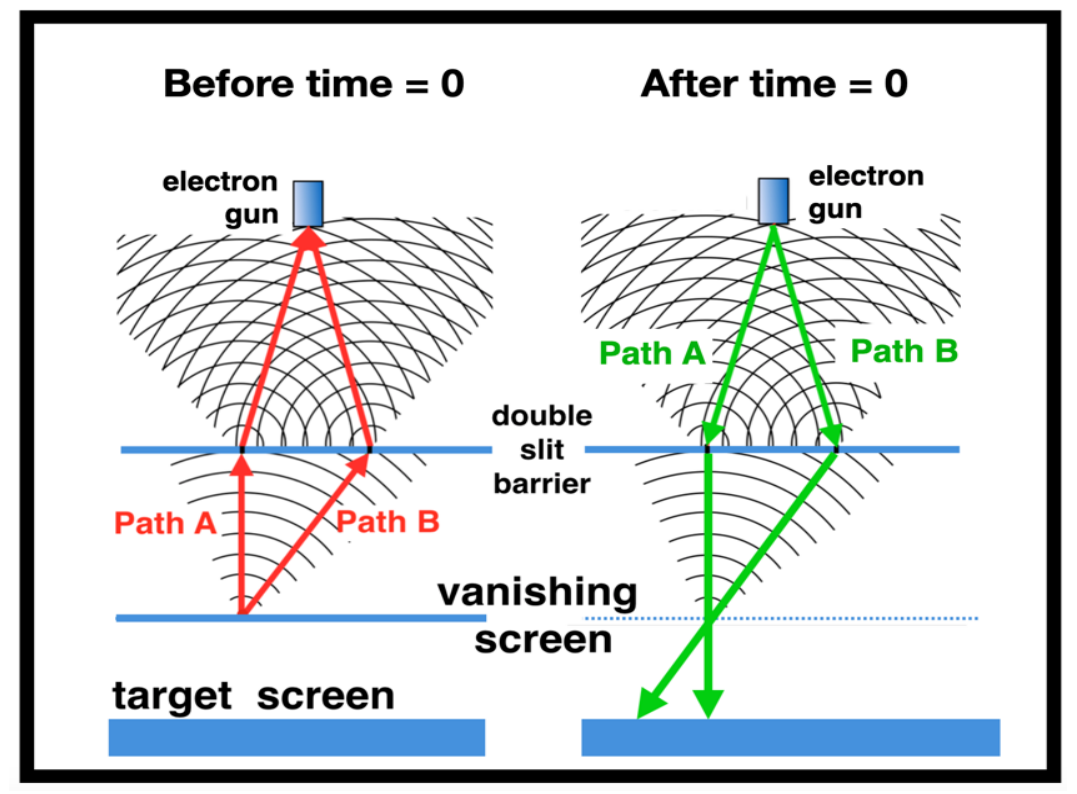

Fig. 28. Here the "vanishing screen" starts opaque then becomes transparent at time $=0$. While it is opaque it emanates elementary waves that interfere in proximity to the particle gun. But several nanoseconds later, when 
a particle is moving southward, the screen is transparent and the particle passes through it and inscribes a wave pattern on the "target screen."

Those electrons are travelling on the green lines in Figure 28. The electrons continue on a straight line until they hit the "target screen" where they inscribe the pattern diagrammed in orange. This is a screwy pattern: not what we expected. For example, in the center of the screen there is a valley (a white area), where we had expected the tallest mountain.

The equipment is designed so nature can vote for one theory or the other. Figure 29 is what will happen if TEW is correct and QM is wrong. If QM is correct and TEW is wrong, then the orange curve will have a familiar shape similar to the blue curve, with the tallest mountain in the center of the screen.

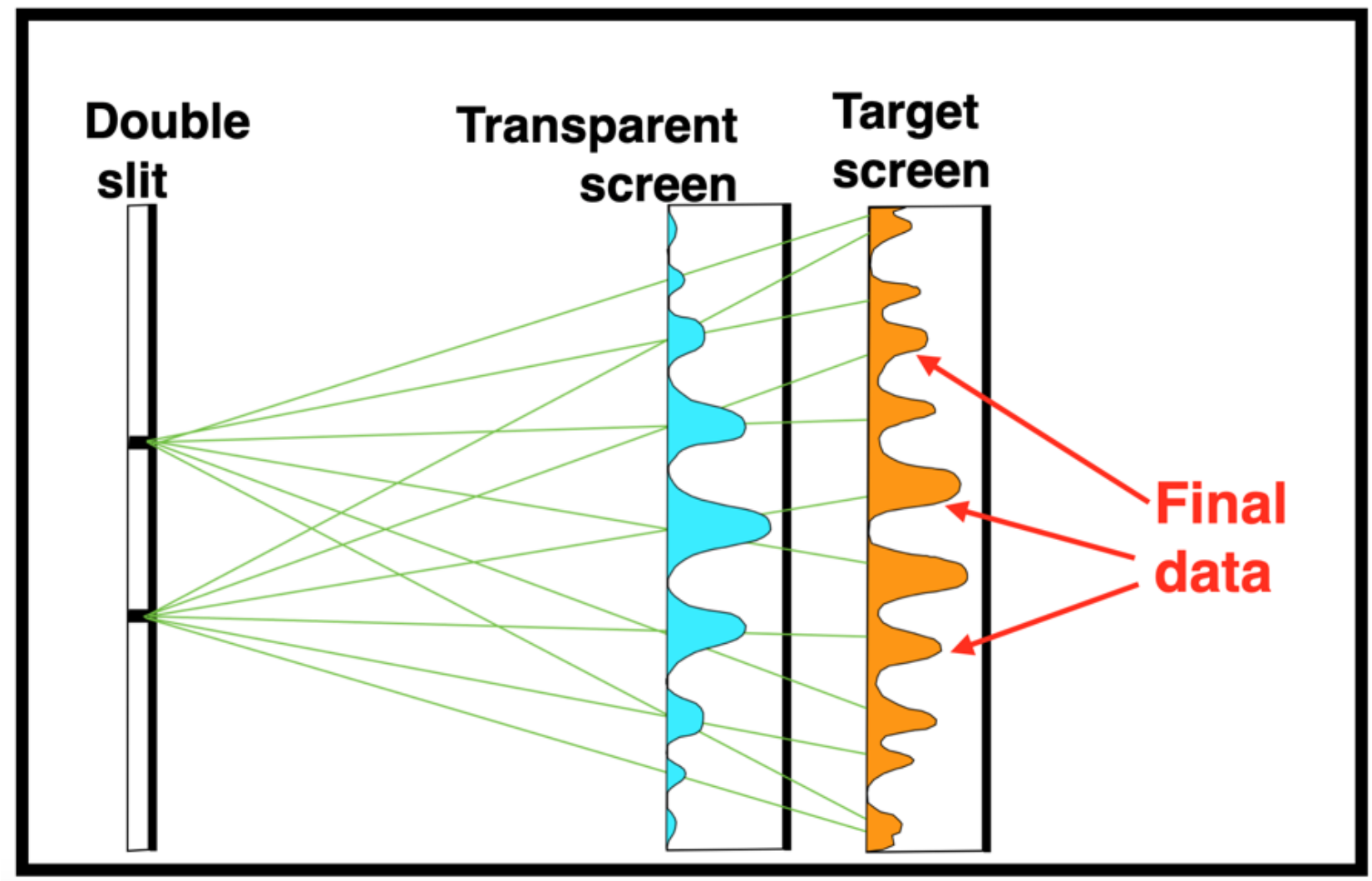

Fig. 29. This is the result of the "vanishing screen" turning from opaque to transparent at time $=0$.

In the next experiment (Figure 30) the "vanishing screen" starts out transparent. Elementary rays start at the target screen (blue-aqua curves) on the far right, pass right through the transparent "vanishing screen" and interfere between the double slit barrier and the electron gun. The electrons that follow these rays backwards are programmed to make the blue curve on the target screen. But once again we have tricked nature. We abruptly insert a barrier that wasn't there before: the vanishing screen is now opaque. When the electrons hit that screen they inscribe a different screwy pattern, as shown in orange in Figure 30. But only if TEW is correct and QM wrong. 


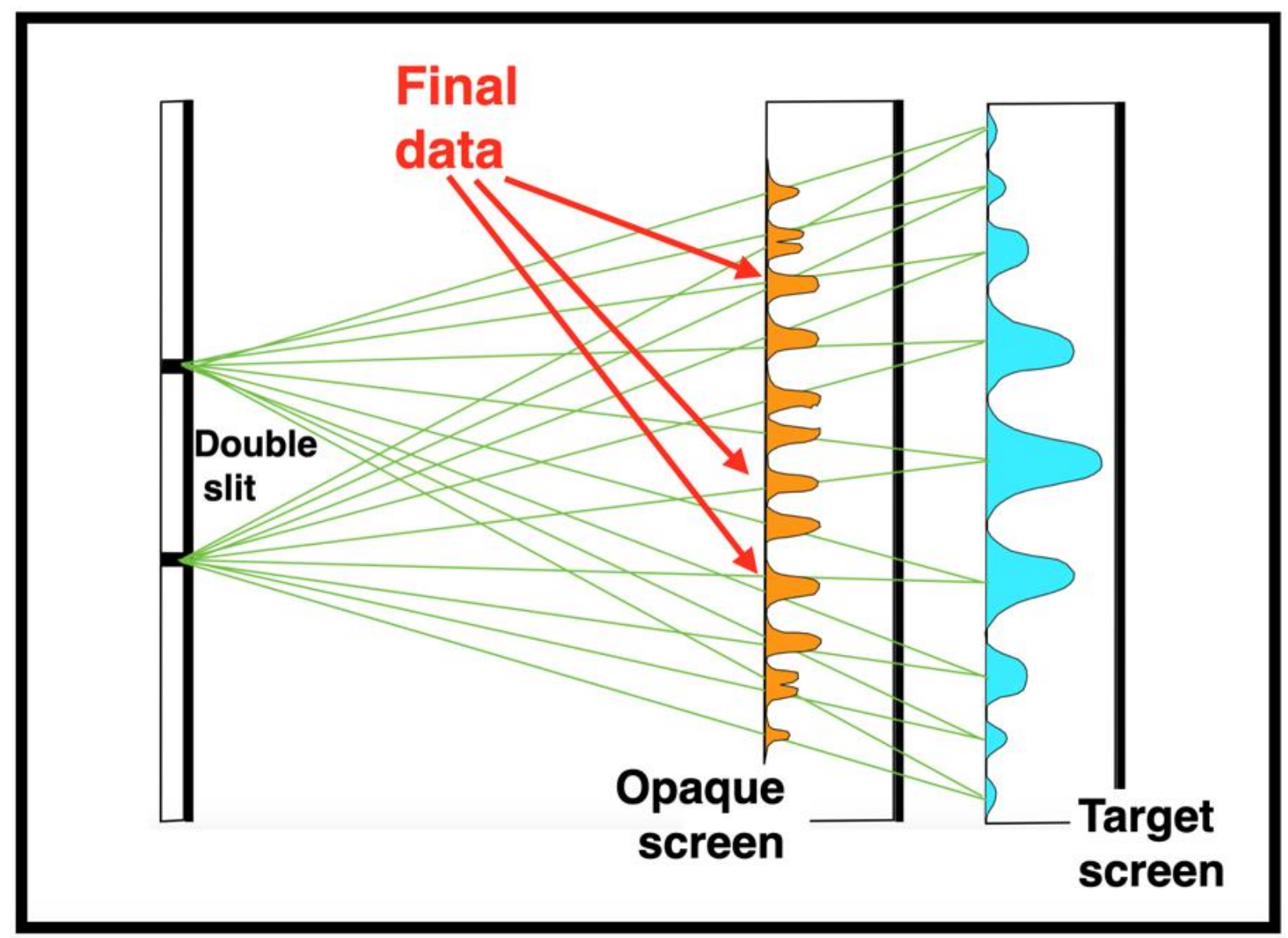

Fig. 30. This is the result of the "vanishing screen" turning from transparent to opaque at time $=0$.

If on the other hand QM is correct and TEW is wrong, then the pattern of the orange curve will look familiar, similar to the blue curve, with the largest mountain in the center of the screen. Thus, nature can vote for one theory or the other.

Caution is needed in designing this equipment. If the experiments produce data that are not what is expected, it could be an error produced because the "vanishing screen" was not transparent to elementary waves at a time when we thought it should be transparent. It is hard to know how to design equipment to study elementary waves, since those waves are previously unknown to science.

\section{Conclusion}

The discovery of elementary waves means that we must rethink everything. For example, it had been taught that electromagnetic waves are self-propagating through the vacuum of space. When we discover that electromagnetic waves are following elementary waves going in the opposite direction and carrying neither magnetism nor energy, it is a fundamental change. Speaking of "fundamental", how many different kinds of elementary waves are there? Is there just one, or are there 17, i.e. one elementary ray for each fundamental particle of the Standard Model of particle physics? That is similar to the question how TEW (conveying zero energy) relates to quantum field theories (full of energy).

As Thomas Kuhn says in The Structure of Scientific Revolutions, paradigm shifts such as the one in this article, tend to be rejected because they sound like gibberish to the old scientific leaders, schooled in the previous paradigm. That is what happened to Alfred Wegener in 1912 when he proposed the existence of an ancient super-continent named Pangaea.(66) His idea sounded unintelligible and was rejected from science. No one could imagine a force strong enough to move continents about. Alfred Holmes proposed a mechanism in 1930, namely seabed spreading due to convection currents in the earth's mantle. That idea also was rejected by the leaders of science because it was a new paradigm that made no sense to the old guard. Yet those ideas of 
Wegener and Holmes inspired young scientists, who researched the age of rock on the seabed, and created the science of plate tectonics, which is the dominant paradigm in geology today. We predict that the future of TEW is similar, namely a paradigm shift led by young people.(56)

The good news is that leaders of science have zero interest in TEW. Why is that good news, instead of bad news? Because this essay and the accompanying video have a goal of encouraging young scientists (teenagers or those under the age of thirty) to take an interest in TEW. Our most valuable gift to young people is to tell them there is a vast and vital arena of science that has been neglected by the experts, and therefore the groundbreaking work waits for a young person to accomplish it.

The leaders of science consider nature to consist of matter and energy and have little interest in something like elementary waves that convey no matter and no energy. Therefore, the leaders of science dismiss TEW as worthless. The fact that elementary waves have been sculpting and shaping nature forever goes unnoticed. This leaves TEW as virgin territory, wide open for new research with a low budget. But if you go in this direction, don't expect your teachers or the experts to encourage you. They will warn you about the drastic mistake you are making to go down this rabbit hole.

It comes down to courage. If you become convinced that wave-particle-duality is false, what price are you willing to pay for your convictions? What risks are you willing to take? The greatest discoveries of science were made by young people who felt scared, unsure of themselves, but who decided to trust their own ideas and plunge into the unknown despite warnings by their teachers. If your opinion is that the experts are wrong when they emphasize that wave particle duality is the cornerstone of science, then perhaps you should listen to yourself, trust your own judgment and act accordingly.

To make this scholarly essay easier to understand, we are producing a lively YouTube video named, " 6 reasons to discard wave particle duality." It will be posted to YouTube a month after this article is published. If you have trouble finding it, search via the author's website ElementaryWave.com.

\section{Acknowledgement}

The author thanks Lewis E. Little.

\section{Conflict of interest}

The author declares that there is no conflict of interest regarding the publication of this article.

\section{Author Biography}

The author is formally trained in mathematics, medicine, world religions and epidemiology. He has degrees from Harvard, Yale, Brown and Case Western Reserve Universities in the United States. He is an ordained clergyman. Over the past half century, the author's work and income have been through his practice of medicine. His passion was to care for indigent patients with severe chronic illnesses. In 2020 he retired. His scholarly work has focused on TEW, a radical revision of QM originally invented by his cousin, Lewis E. Little. Boyd was sole author of two dozen scholarly articles on TEW published in peer reviewed journals of physics, math and chemistry. His website is ElementaryWave.com.

The author feels called to promote TEW as a solution to the intellectual and spiritual muddle produced by QM. Until now he has avoided discussing his spiritual commitments because so much of science is controlled by scholars who are fiercely opposed to a religious perspective. If you mention spirituality, many of them immediately assume you are narrow minded and bigoted. However, when the author looks at the big picture it is those anti-religious scientists who are narrow minded and bigoted. The world needs scientists who have the courage to declare what motivates them to serve humanity by promoting science. The author's scientific compass is defined by these words from the Bible: "You will know the truth, and the truth will set you free" (Gospel of John 8:32). 


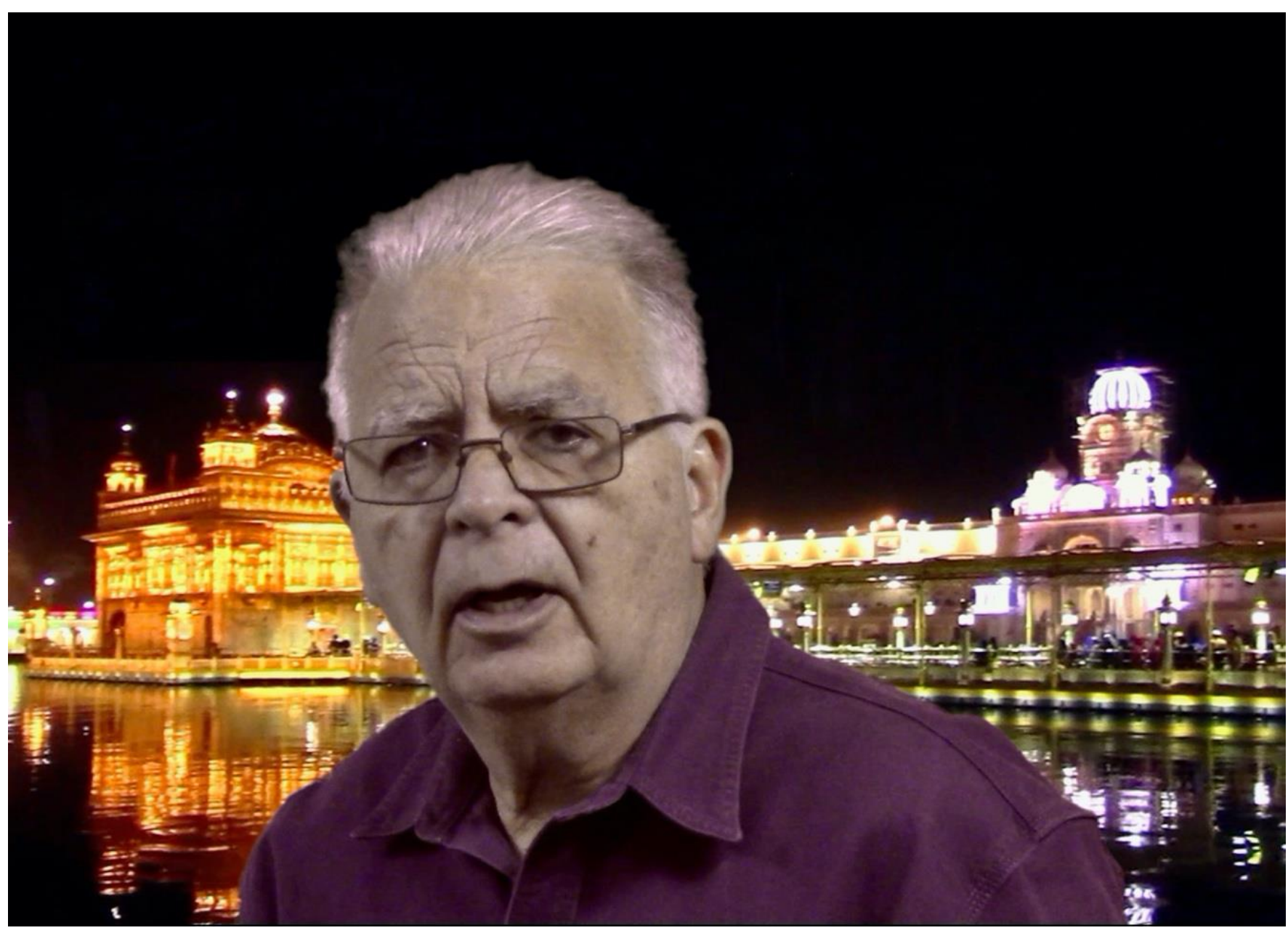

Jeffrey H. Boyd, 2021, has never been to India. Now, hemmed in by COVID-19

he rarely leaves his house, except in his imagination, as in this picture. His

primary commitment is to God. That is the goal and purpose of his life.

\section{References}

1. Aspect, Bell's inequality test: more ideal than ever. Nature 398, 189-190 (1999). DOI: 10.1038/18296

2. Aspect, P. Grangier, and G. Roger. Experimental realization of Einstein-Podolsky-Rosen-Bohm Gedanken- experiment. Physical Review Letters, 49, 91-94, 1982. DOI: 10.1103/PhysRevLett.49.91

3. Aspect, P. Grangier, and G. Roger. Experimental tests of realistic local theories via Bell's theorem. Physical Review Letters, 47, 460-463, 1981. DOI: 10.1103/PhysRevLett.47.460

4. J. Baggott, The Quantum Story, Oxford University Press, 2011. ISBN:978-0-19-956684-6

5. A. Becker, What Is Real? Basic Books, 2018. ISBN:978-0-19-956684-6

6. J. S. Bell. On the Einstein Podolsky Rosen paradox. Physics 1, 195-200, 1964. DOI: 10.1103/PhysicsPhysiqueFizika.1.195

7. J. S. Bell, "Bertlmann's socks and the nature of reality, Journal de Physique, vol. 42, 1981, pp. C2-C41. DOI: 10.1051/jphyscol:1981202

8. J. S. Bell, "The paradox of Einstein, Podolsky and Rosen: action at a distance in quantum mechanics?," Speculations in Science and Technology, vol. 10, pp. 269-285 (1987).

9. H. Bernien, A. E. Dréau, A. Reiserer, et. al., "Loophole-free Bell inequality violation using electron spins separated

by

1.3

kilometres,"

Nature

(2015) 
http://www.nature.com/nature/journal/vaop/ncurrent/full/nature15759.html

DOI: 10.1038/nature15759

10. M. Born, "Zur Quantenmechanik der Stoßvorgänge. Zeitschrift für Physik, 37," pp. 863-867, 1926. DOI: 10.1007/BF01397477

11. M. Born, "On the quantum mechanics of collisions," in J. A. Wheeler and W. H. Zurek (eds.), Quantum Theory and Measurement, Princeton, pp.50-55, 1983. ISBN 978-0-691-08316-2.

12. J. H. Boyd, "The Periodic Table needs negative orbitals in order to eliminate quantum weirdness," Journal of Advances in Chemistry, vol. 17, pp.88-125, 2020. DOI: 10.24297/jac.v17i.8865

13. J. H. Boyd, "There are two solutions to the equations of Feynman's Quantum Electrodynamics (QED)," Journal of Advances in Physics, vol. 18, pp.39-57, 2020. DOI: 10.24297/jap.v18i.8831.

14. J. H. Boyd, "If the propagator of QED were reversed, the mathematics of Nature would be much simpler," Journal of Advances in Mathematics, vol. 18, pp. 129-153, 2020. DOI: 10.24297/jam.v18i.8746

15. J. H. Boyd, "A tiny, counterintuitive change to the mathematics of the Schrödinger wave packet and Quantum ElectroDynamics could vastly simplify how we view Nature," Journal of Advances in Physics, vol. 17, pp. 169-203, 2020. DOI: 10.24297/jap.v17i.8696

16. J. H. Boyd, "New Schrödinger wave mathematics changes experiments from saying there is, to denying there is quantum weirdness," Journal of Advances in Mathematics, vol. 18, pp. 82-117, 2020. DOI: 10.24297/jap.v17i.8696

17. J. H. Boyd, "Decrypting the central mystery of quantum mathematics: Part 3. A non-Einstein, non-QM view of Bell test experiments," Journal of Advances in Mathematics, vol. 17, pp. 315-331, 2019. DOI: $10.24297 / j a m . v 17 i 0.8490$

18. J. H. Boyd, "The quantum world is astonishingly similar to our world," Journal of Advances in Physics, vol. 14, pp. 5598-5610, 2018. DOI: 10.24297/jap.v14i2.7555

19. J. H. Boyd, "A paradigm shift, Part 2: A new local realism explains Bell test," Journal of Advances in Mathematics, vol. 10, pp. 3828-3839, 2015. DOI: 10.24297/jam.v10i9.1884

20. J. H. Boyd, "A paradigm shift, Part 4: Quantum computers and the local realism of all 4 Bell states," Journal of Advances in Mathematics, vol. 11, pp. 5476-5493, 2015. DOI: 10.24297/jam.v11i7.1224

21. J. H. Boyd, "Re-thinking Alain Aspect's 1982 Bell test experiment with delayed choice," Physics Essays, vol. 26, pp. 582-591, 2013. DOI: 10.4006/0836-1398-26.1.100 10.4006/0836-1398-26.4.582

22. J. H. Boyd, "Decrypting the central mystery of quantum mathematics: Part 1. The double slit experiment," Journal of Advances in Mathematics, 2 vol. 17, pp. 255-282, 2019. DOI: 10.24297/jam.v17i0.8475

23. J. H. Boyd, "Decrypting the central mystery of quantum mathematics: Part 2. A mountain of empirical data supports TEW," Journal of Advances in Mathematics, vol. 17, pp. 283-314, 2019. DOI: 10.24297/jam.v17i0.8489

24. J. H. Boyd, "Decrypting the central mystery of quantum mathematics: Part 3. A non-Einstein, non-QM view of Bell test experiments," Journal of Advances in Mathematics, vol. 17, pp. 315-331, 2019. DOI: 10.24297/jam.v17i0.8490

25. J. H. Boyd, "Decrypting the central mystery of quantum mathematics: Part 4. In what medium do Elementary Waves travel?" Journal of Advances in Mathematics, vol. 17, pp. 332-351, 2019. DOI: 10.24297/jam.v17i0.8491

26. J. H. Boyd, "The quantum world is astonishingly similar to our world," Journal of Advances in Physics, vol. 14, 5598-5610, 2018. DOI: 10.24297/jap.v14i2.7555

27. J. H. Boyd, "The von Neumann and double slit paradoxes lead to a new Schrödinger wave mathematics," Journal of Advances in Physics, vol.14, 5812-5834, 2018. doi.org/10.24297/jap.v14i3.7820 
28. J. H. Boyd, "The Boyd Conjecture," Journal of Advances in Physics, vol. 13, 4830-4837, 2017. DOI: 10.24297/jap.v13i4.6038

29. J. H. Boyd, "A symmetry hidden at the center of quantum mathematics causes a disconnect between quantum math and quantum mechanics," Journal of Advances in Mathematics, vol. 13, 7379-7386, 2017. DOI: 10.24297/jam.v13i4.6413

30. J. H. Boyd, "Paul Dirac's view of the Theory of Elementary Waves," Journal of Advances in Physics, vol. 13, 4731-4734, 2017. DOI: 10.24297/jap.v13i3.5921

31. J. H. Boyd, "A paradigm shift, Part 1: The Theory of Elementary Waves (TEW)," Journal of Advances in Mathematics, vol. 10, 3828-3839, 2015. DOI: 10.24297/jam.v10i9.1908

32. J. H. Boyd, "A paradigm shift, Part 2: A new local realism explains Bell test," Journal of Advances in Mathematics, vol. 10, 3828-3839, 2015. DOI: 10.24297/jam.v10i9.1884

33. J. H. Boyd, "A paradigm shift, Part 3: A mirror image of Feynman's quantum electrodynamics (QED)," Journal of Advances in Mathematics, vol. 11, 3977-3991, 2015. DOI: 10.24297/jam.v11i2.1283

34. J. H. Boyd, "A paradigm shift, Part 4: Quantum computers and the local realism of all 4 Bell states," Journal of Advances in Mathematics, vol. 11, 5476-5493, 2015. DOI: 10.24297/jam.v11i7.1224

35. J. H. Boyd, "The Theory of Elementary Waves eliminates wave-particle-duality," Journal of Advances in Physics, vol. 7, 1916-1922, 2015. DOI: 10.24297/jap.v7i3.1576

36. J. H. Boyd, "A new variety of local realism explains a Bell test experiment," Journal of Advances in Physics, vol. 8, 2051-2058, 2015. DOI: 10.24297/jap.v8i1.1541

37. J. H. Boyd, "A proposed physical analog of a quantum amplitude," Journal of Advances in Physics, vol. 10, pp.2774-2783, 2015. DOI: 10.24297/jap.v10i3.1324

38. J. H. Boyd, "Re-thinking a delayed choice quantum eraser experiment," Physics Essays, vol. 26, pp.100109, 2013. DOI: 10.4006/0836-1398-26.1.100

39. J. H. Boyd, "Re-thinking Alain Aspect's 1982 Bell test experiment with delayed choice," Physics Essays, vol. 26, pp.582-591, 2013. DOI: 10.4006/0836-1398-26.1.100 10.4006/0836-1398-26.4.582

40. J. H. Boyd, "Rethinking a Wheeler delayed choice gedanken experiment," Physics Essays, vol. 25, pp.390396, 2012. DOI: 10.4006/0836-1398-25.3.390

41. J. F. Clauser, M. A. Horne, A. Shimony and R. A. Holt, "Proposed experiment to test local hidden-variable theories." Physical Review Letters 23, 880-884, 1969. DOI: 10.1103/PhysRevLett.23.880

42. B. J. Davisson and L. Germer, "Reflection of electrons by a crystal of nickel," Nature, vol. 119, pp.558-560, 1927. DOI: $10.1038 / 119558 \mathrm{a} 0$

43. B. J. Davisson, "The diffraction of electrons by a crystal of nickel," Bell System Technical Journal, vol. 7, pp. 90-105, 1928. DOl: 10.1002/j.1538-7305.1928.tb00342.x

44. B. J. Davisson, "Are electrons waves?" Franklin Institute Journal, vol. 205, 597, 1928. DOI: 10.1016/S00160032(28)90979-5

45. R. P. Feynman, Feynman Lectures on Physics, vol. 2. New York: Basic Books, c1964. ISBN-13: 978-04650294-0

46. R. P. Feynman and A. R. Hibbs, Quantum Mechanics and Path Integrals, Mineola, NY: Dover Publications, C1965. ISBN-13 978-0-468-47722-0.

47. G. Gabrielse, D. Hanneke, T. Kinoshita, et. al., "New determination of the fine structure constant from the electron g value and QED," Physical Review Letters, 97, 030802 (2006). DOI: 10.1007/BF00670751

48. R. Galchen and D.Z. Albert, "Was Einstein wrong?" Scientific American, vol. 300, pp. 32-39, 2009. DOI: 10.1038/scientificamerican0309-32. 
49. M. Giustina, M.A.M. Versteegh, S. Wengerowsky, et. al. "Significant-loophole-free test of local realism with entangled photons." Physical Review Letters 115, 250401 (2015). DOI: 10.1103/PhysRevLett.115.250401

50. M. Giustina, "Significant-loophole-free test of local realism with entangled photons." Rotman Institute of Philosophy, 2016. https://www.youtube.com/watch?v=tgoWM4Jcl-s

51. M. Giustina, M.A.M. Versteegh, S. Wengerowsky, et. al. "Significant-loophole-free test of local realism with entangled photons." Archiv.org. 2015. arXiv:1511.03190v2.

52. A. Giustina, A. Mech, S. Ramelow, et. al., "Bell violation using entangled photons without the fairsampling assumption," Nature, vol. 497, pp. 227-230, 2013. DOI: 10.1038/nature12012

53. B. Hensen, H. Bernien, A. E. Dréau, et.al., "Loophole-free Bell inequality violation using electron spins separated by 1.3 kilometres," Nature 526, (2015), DOI: 10.1038/nature15759.

54. J. Hofmann, M. Krug, N. Ortegel, et. al., "Heralded entanglement between widely separated atoms," Science 337, 72-75 (2012). DOI: 10.1126/science.1221856

55. H. Kaiser, R. Clothier, S.A. Werner, H. Rauch and H. Wölwitsch, "Coherence and spectral filtering in neutron interferometry," Physical Review A, vol. 45, 31-42, 1992. DOI: 10.1103/PhysRevA.45.31

56. T. S. Kuhn, The Structure of Scientific Revolutions, Chicago: University of Chicago Press, 1970. ISBN 9780-226-45803-8.

57. P. G. Kwiat, K. Mattle, H. Weinfurter, et. al., "New high-intensity source of polarization-entangled photon pairs," Physical Review Letters, 75 (\#24) pp. 4337-4341 (1995). DOI: 10.1103/PhysRevLett.75.4337

58. B. Lim, "Bell test: disproving local realism," 2017. https://www.youtube.com/watch? $v=U b N m V 9 C W b H 0$

59. L. E. Little, "Theory of Elementary Waves," Physics Essays, vol. 9, pp.100-134, 1996. https://doi.org/10.4006/1.3029212

60. N. D. Mermin, "Is the moon there when nobody looks? Reality and the quantum theory," Physics Today, 38, 38-47 (1985). DOI: 10.1063/1.880968

61. W. A. Miller and J. A. Wheeler, "Delayed choice experiments and Bohr's elementary quantum phenomenon," in ed. S. Makefuchi, Proceedings of the International Symposium Foundations of Quantum Mechanics, (Hitachi Ltd., Kokubunji, Tokyo 1983), pp.140-152.

62. R. L. Pfleegor and L. Mandel, "Interference of independent photon beams," Physical Review, vol. 159, pp.1084-1088, 1967. DOI: 10.1103/PhysRev.159.1084

63. R. L. Pfleegor and L. Mandel, "Further experiments on interference of independent photon beams at low light levels," Journal of the Optical Society of America, vol. 58, pp.946-950, 1968. DOI: 10.1364/JOSA.58.000946

64. R. Resnick, "Quantum Mechanics 10b Bell's inequality," ViaScience, 2014. https://www.youtube.com/watch?v=8UxYKN1q5sl

65. C. Salart, A. Baas, J. A. W. van Houwelingen, N. Gisin, and H. Zbinden, "Spacelike separation in a Bell test assuming gravitationally induced collapses," Physical Review Letters 100, 220404-1 to 220404-4 (2008). DOI: 10.1103/PhysRevLett.100.220404

66. A. Wegener, The Origin of Continents and Oceans, translated by John Biram, New York, Dover Publications Inc.1966, Library of Congress Catalog Card Number: 66-28270.

67. G. Weihs, T. Jennewein, C. Simon, et. al., "Violation of Bell's inequality under strict Einstein locality conditions," Physical Review Letters 81, 5039 (1998). DOI: 10.1103/PhysRevLett.81.5039

68. A. Yurke and B. Stoler, Einstein-Podolsky-Rosen effects from independent particle sources, Physical Review Letters 68, 1251-1254 (1992). DOI: 10.1103/PhysRevLett.68.1251

69. S. A. Werner, R. Clothier, H. Kaiser, et.al., "Spectral filtering in neutron interferometry," Physical Review Letters, vol. 67, pp.683-686, 1991. DOI: 10.1103/PhysRevLett.67.683

70. M. Żukowski, Zeilinger, A., Horne, M. A. \& Ekert, A. K. "Event-ready-detectors" Bell experiment via entanglement swapping. Physical Review Letters 71, 4287-4290 (1993). DOI: 10.1103/PhysRevLett.71.4287 